\title{
Evolution of the Suprathermal Proton Population at Interplanetary Shocks
}

\author{
D. Lario ${ }^{1,5}(1)$, L. Berger $^{2}$, R. B. Decker ${ }^{1}$, R. F. Wimmer-Schweingruber ${ }^{2}$ (1), L. B. Wilson, $\mathrm{III}^{3}{ }^{1}$, J. Giacalone ${ }^{4}$, and E. C. Roelof ${ }^{1}$ \\ ${ }^{1}$ The Johns Hopkins University, Applied Physics Laboratory, 11100 Johns Hopkins Rd., Laurel, MD 20723, USA; david.larioloyo@nasa.gov \\ ${ }^{2}$ Institut für Experimentelle und Angewandte Physik (IEAP), Christian-Albrechts-Universität zu Kiel Leibnizstrasse 11, D-24118 Kiel, Germany \\ ${ }^{3}$ NASA, Goddard Space Flight Center, Heliophysics Science Division, 8800 Greenbelt Rd., Greenbelt, MD 20771, USA \\ ${ }^{4}$ Lunar and Planetary Laboratory, University of Arizona, 1629 E. University Blvd., Tucson, AZ 85721, USA \\ Received 2018 August 15; revised 2019 April 12; accepted 2019 April 29; published 2019 June 11
}

\begin{abstract}
We investigate the evolution of the suprathermal (ST) proton population as interplanetary shocks cross 1 au. The variability of the ST proton intensities and energy spectra upstream of the shocks is analyzed in terms of the shock parameters, upstream magnetic field configurations, and preexisting upstream populations. Propitious conditions for the observation of ST particles at distances far upstream from the shock occur in parallel shock configurations when particles can easily escape from the shock vicinity. In this situation, ST intensity enhancements show onsets characterized by velocity dispersion effects and energy spectra that develop into a "hump" profile peaking around $\sim 10 \mathrm{keV}$ just before the arrival of the shock. The observation of field-aligned proton beams at low energies $(5-10 \mathrm{keV})$ is possible under conditions that facilitate the scatter-free propagation of the particles streaming out of the shock. Upstream of perpendicular shocks, ST intensity enhancements are only observed in close proximity to the shock. Power-law proton spectra develop downstream of the shocks. The functional form for the downstream phase-space density proportional to $v^{-5}$ is observed only over a limited range of ST energies. The absence of ST populations observed far upstream of interplanetary shocks raises questions about whether ST protons contribute as a seed particle population in the processes of particle acceleration at shocks.
\end{abstract}

Key words: acceleration of particles - interplanetary medium - shock waves - solar wind

\section{Introduction}

Enhancements of energetic particle intensities associated with the passage of interplanetary (IP) shocks have been historically termed energetic storm particle (ESP) events (Bryant et al. 1962). Energetic particles in ESP events result from processes of particle acceleration at shocks (e.g., Lee 2005; Giacalone 2012, and references therein) and/or in the compressed turbulent region formed behind the shocks (e.g., van Nes et al. 1985; Fisk \& Gloeckler 2012; Zank et al. 2015). The acceleration of energetic particles may start as soon as the shocks form close to the Sun and continue as the shocks pass the spacecraft (e.g., Lario et al. 1998, 2017). Scattering by self-excited magnetic field fluctuations upstream of the shock, as well as by field fluctuations in the turbulent medium downstream of the shock, have been suggested as mechanisms necessary for the efficient confinement of particles in the shock vicinity (e.g., Lee 1983, 2005). The observation of these previously accelerated particles, together with particles accelerated at the time of the shock passage, constitute the intensity enhancement seen in ESP events.

The origins of the seed particle populations for the particle acceleration mechanisms at traveling shocks are still under debate. Possible origins include thermal solar wind particles, preexisting suprathermal (ST) ions, and/or a mixture of both (e.g., Desai et al. 2016; Desai \& Giacalone 2016, and references therein). The efficiency of a given shock in accelerating particles is determined not only by the properties of the shock but also by the nature of the upstream particle distributions that the traveling shock encounters (e.g., Giacalone 2005). The efficiency of particle acceleration in the downstream region of the shocks also depends on the plasma properties of that region (e.g., the

\footnotetext{
5 Now at NASA, Goddard Space Flight Center, Heliophysics Science Division, 8800 Greenbelt Rd., Greenbelt, MD 20771, USA.
}

existence of solar wind compressions; Fisk \& Gloeckler 2012). The lower the energy of the particles, the more prominent the ESP enhancements observed around shocks (Lario et al. 2003). Therefore, the characterization of the particle populations, from thermal to high energies, upstream and downstream of the shocks, is required in order to determine the seed particle populations and the effects that the shocks produce on these populations.

In this paper, we analyze the evolution of the ST and highenergy proton populations across IP shocks. We denote the ST population as those protons that, when plotting particle intensities as a function of energy, form a tail at energies above the quasi-Gaussian function that best describes the thermal component (usually above a few keV). By energetic population, we denote those protons detected by energetic particle instrumentation (usually above $\sim 50 \mathrm{keV}$ ). Although artificial, this distinction allows us to discuss the capability of space instrumentation to measure both populations during the passage of IP shocks. In this paper, we analyze the continuity of the energy spectra between the ST regime (at energies $\lesssim 50 \mathrm{keV}$ ) and the energetic particle regime (at energies $\gtrsim 50 \mathrm{keV}$ ) for IP shocks measured at $\sim 1$ au from the Sun.

Prior attempts to analyze the evolution of the ST proton population and its relationship with high-energy populations in transient IP shocks have been very limited. Frank (1970) was the first to report IP proton energy spectra in the energy range $5 \mathrm{keV} \lesssim E \lesssim 50 \mathrm{keV}$ upstream of shocks showing a broad energy distribution with a peak around $\sim 15 \mathrm{keV}$ and diminishing toward low $(\sim 6 \mathrm{keV})$ and high $(\sim 40 \mathrm{keV})$ energies (see Figure 2 in Frank 1970). Gosling et al. (1981) combined data from two different instruments on board the International Sun-Earth Explorers ISEE-2 and ISEE-3 to construct proton energy spectra from thermal energies to $\sim 1.6 \mathrm{MeV}$ in the downstream region of an IP shock. The smooth continuity of 
the energy spectrum was suggested as evidence of an ESP population being accelerated directly out of the solar wind thermal population or from a quiescent ST tail. However, Gosling et al. (1981) did not discuss the evolution of these populations prior to the shock passage.

Later, Gosling et al. (1984) used ISEE-I and ISEE-3 instrumentation to study the presence of upstream ions at IP shocks at energies below $\sim 35 \mathrm{keV}$. They found that only seven out of the 17 IP shocks studied had detectable upstream shockassociated STs, and only three of them had intensities that were sufficient to perform quantitative analysis. The intensity of upstream ST ions did not appear to be controlled by the local values of either the magnetosonic Mach number $\left(M_{\mathrm{ms}}\right)$ or the angle between the shock normal and the upstream magnetic field $\left(\theta_{B n}\right)$, though the shocks for which ST ions were not detected had $M_{\mathrm{ms}} \lesssim 1.6$. For the three events with high enough intensities, the observed flux of upstream ions increased monotonically closer to the shock, and the distributions were roughly isotropic in the solar wind frame, with the exception of one event in which field-aligned flow away from the shock was observed, although the beam did not exhibit a well-defined peak in velocity space clearly separated from the solar wind beam (see Figure 5 in Gosling et al. 1984). The connection between ion populations below $\sim 35 \mathrm{keV}$ and higher-energy ion populations was not established.

Ogilvie et al. (1993) used ion data from the Solar Wind Ion Composition Spectrometer (SWICS) on board the Ulysses spacecraft (Gloeckler et al. 1992) in the ecliptic plane at heliocentric distances between 1.33 and 3.14 au to determine the presence of high-velocity tails in the velocity distribution of protons. These tails extended up to $\sim 1200 \mathrm{~km} \mathrm{~s}^{-1}$ in the spacecraft frame of reference (i.e., $\sim 7.5 \mathrm{keV}$ ) and were observed following the passage of shocks. At these energies, the proton distributions were nearly isotropic in the spacecraft frame. These authors stated that there was no detailed correspondence between the presence of ST tails and intense solar energetic particle (SEP) fluxes at energies $\gtrsim 50 \mathrm{keV}$, concluding that ST tails in the downstream region of shocks were not due to an increase of SEP fluxes but rather to acceleration from the ambient solar wind.

Tokar et al. (2000) studied the evolution of $\sim 700 \mathrm{eV} / \mathrm{q}$ to $\sim 3 \mathrm{keV} / \mathrm{q}$ ions in an IP shock observed by the Solar Wind Electron, Proton, and Alpha Monitor (SWEPAM; McComas et al. 1998) on board the Advanced Composition Explorer $(A C E)$ to determine the presence of an upstream proton fieldaligned beam at energies $\lesssim 2.5 \mathrm{keV}$. The connection with higher-energy particles was established just by confirming that this shock also exhibited ion intensity enhancements up to $\sim 100 \mathrm{keV}$, but the spectral transition from $\sim 3$ to $\sim 45 \mathrm{keV}$ was not studied.

Fisk \& Gloeckler (2012) studied the evolution of ST tails around IP shocks measured by the SWICS instrument on board $A C E$ (Gloeckler et al. 1998). They integrated proton intensities over the speed range from $\sim 500$ to $2000 \mathrm{~km} \mathrm{~s}^{-1}$ and used $1 \mathrm{hr}$ averages to show that, downstream of IP shocks and/or when solar wind compression regions are formed, the intensity of the ST tails enhances, and, only when the ST tail density is high, the energy spectra of the differential flux $j(E)$ tend to be a power law close to $E^{-1.5}$ (or $v^{-5}$ when expressed in terms of the phase-space density $f(v)$ ). Ahead of the shock, the energy spectra might exhibit complex forms with multiple components that might result from local acceleration of particles but also from their transport from downstream of the shocks to upstream (Fisk \& Gloeckler 2014). However, a detailed discussion of the evolution of the ST population from upstream to downstream was not offered.

Finally, Kajdič et al. (2017) used measurements from the two ARTEMIS satellites orbiting the Moon to identify different types of ion distributions that may exist upstream of a single IP shock (in analogy with those usually observed upstream of the Earth's bow shock; e.g., Gosling et al. 1978; Thomsen 1985). Field-aligned beams were observed upstream of an IP shock ( $\sim 60$ minutes before the shock crossing) with energies that extended from $\sim 20$ to $\sim 190 \mathrm{keV}$ in the spacecraft frame of reference. As the shock approached the spacecraft, the ion distributions intensified and became more diffuse around the magnetic field direction (see Figure 3 in Kajdič et al. 2017). Gosling (1983) suggested that the observation of field-aligned beams of low-energy ions upstream of IP shocks, such as the one reported by Kajdič et al. (2017), is quite difficult. The large extension of IP shocks allows spacecraft to establish magnetic connection with the shock for long-lasting periods. Ion distributions in ESP events are constituted not only of particles locally accelerated but also of particles accelerated at earlier stages. Particles leaving the shock may encounter and be scattered by magnetic perturbations generated further upstream, resulting in diffuse distributions (as opposite to field-aligned beams) that are usually observed in ESP events (Gosling 1983; Wilson et al. 2009; Blanco-Cano et al. 2016).

Here we report measurements of ST and high-energy protons by the $A C E$ and Wind spacecraft during the passage of IP shocks. The observation of these populations depends on the capability of the instruments on board these spacecraft to detect particle populations of a given intensity and angular distribution. The sensitivity and fields of view of the instruments are not always capable of discerning the different types of particle velocity-space distributions that may exist upstream of IP shocks (e.g., Gosling 1983; Kajdič et al. 2017), but they allow us to distinguish how an ST population evolves during the passage of an IP shock. We distinguish (1) shocks for which the ST population intensifies first at high energies and later at lower energies nearer the shock showing velocity dispersion, (2) shocks for which the intensity increase is only observed at low energies $(\lesssim 10 \mathrm{keV})$, and (3) shocks for which there is no significant enhancement of the ST population before the shock passage. In case (1), we use the term "velocity dispersion" in the sense that an upstream ion distribution that may be quasisteady in the shock frame with high energies extending further upstream of the shock will, when viewed in the spacecraft frame, appear to show velocity dispersion as the spacecraft penetrates further into the pre-shock region toward the shock. We discuss how these different types of ST evolution depend on both the large-scale magnetic field configuration upstream of the shock and the ability of the particles to escape from the vicinity of the shock.

\section{Observations}

We use energetic particle data from the $A C E$ and Wind spacecraft. A spin-stabilized spacecraft with the spin axis pointing toward the Sun to within $\pm 20^{\circ}, A C E$ occupies a halo orbit about the L1 Earth-Sun liberation point. Wind is a spinstabilized spacecraft with the spin axis pointing toward the south ecliptic pole. During the time intervals analyzed in this article, Wind, which moved throughout a variety of positions 
Table 1

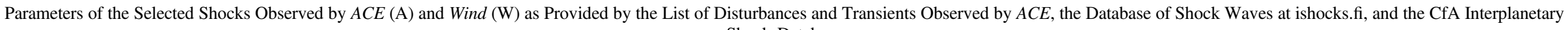
Shock Database

\begin{tabular}{|c|c|c|c|c|c|c|c|c|c|c|c|c|c|c|c|}
\hline \multirow{2}{*}{$\begin{array}{c}\text { Shock Time } \\
\text { (SC Year/Day/UT) [doy] }\end{array}$} & \multicolumn{5}{|c|}{$A C E$ Transient List ${ }^{\mathrm{a}}$} & \multicolumn{5}{|c|}{ ipshocks.fif ${ }^{\mathrm{b}}$} & \multicolumn{5}{|c|}{ CfA Shock List $(\mathrm{RH} 08)^{\mathrm{c}}$} \\
\hline & $r_{n}$ & $r_{b}$ & $V_{s}$ & $M_{\mathrm{A}}$ & $\theta_{B n}$ & $r_{n}$ & $r_{b}$ & $V_{s}$ & $M_{\mathrm{ms}}$ & $\theta_{B n}$ & $r_{n}$ & $r_{b}$ & $V_{s}$ & $M_{\mathrm{ms}}$ & $\theta_{B n}$ \\
\hline $\begin{array}{l}\text { A } 2001 / 023 / 10: 06: 36 \\
\text { [023.4212] }\end{array}$ & $3.1 \pm 1.6$ & $1.0 \pm 0.8$ & $532 \pm 32$ & $2.8 \pm 1.0$ & $03 \pm 41$ & $4.4 \pm 0.8$ & $1.8 \pm 0.5$ & $418 \pm 357$ & $1.9 \pm 1.9$ & $56 \pm 52$ & $5.0 \pm 0.8$ & $1.4 \pm 0.6$ & $582 \pm 17$ & $2.9 \pm 0.3$ & $19 \pm 08$ \\
\hline $\begin{array}{l}\text { W 2001/023/10:49:36 } \\
\text { [023.4511] }\end{array}$ & $\cdots$ & $\cdots$ & $\cdots$ & $\cdots$ & $\cdots$ & $1.8 \pm 2.0$ & $2.3 \pm 0.5$ & $672 \pm 379$ & $3.6 \pm 4.8$ & $22 \pm 16$ & $3.2 \pm 0.4$ & $2.4 \pm 0.1$ & $615 \pm 18$ & $3.2 \pm 0.3$ & $32 \pm 03$ \\
\hline $\begin{array}{l}\text { A } 2002 / 198 / 15: 26: 14 \\
\text { [198.6432] }\end{array}$ & $2.6 \pm 0.2$ & $2.2 \pm 0.6$ & $493 \pm 40$ & $2.2 \pm 0.4$ & $35 \pm 12$ & $2.8 \pm 0.7$ & $2.5 \pm 0.3$ & $524 \pm 72$ & $2.0 \pm 0.7$ & $16 \pm 35$ & $2.7 \pm 0.5$ & $2.5 \pm 4.4$ & $379 \pm 16$ & $0.8 \pm 0.1$ & $62 \pm 21$ \\
\hline $\begin{array}{l}\text { W 2002/198/15:55:33 } \\
\quad[198.6636]\end{array}$ & $\cdots$ & $\cdots$ & $\cdots$ & $\cdots$ & $\cdots$ & $3.8 \pm 0.7$ & $2.2 \pm 0.3$ & $407 \pm 290$ & $1.0 \pm 1.5$ & $64 \pm 84$ & $3.1 \pm 0.7$ & $2.3 \pm 0.2$ & $505 \pm 18$ & $2.2 \pm 0.1$ & $29 \pm 10$ \\
\hline $\begin{array}{l}\text { A } 2001 / 304 / 12: 53: 12 \\
\text { [304.5369] }\end{array}$ & $2.1 \pm 0.3$ & $1.7 \pm 0.9$ & $458 \pm 58$ & $2.7 \pm 0.7$ & $39 \pm 25$ & $2.4 \pm 0.6$ & $1.9 \pm 0.1$ & $455 \pm 24$ & $2.0 \pm 0.4$ & $19 \pm 05$ & $2.5 \pm 0.7$ & $1.9 \pm 0.2$ & $447 \pm 28$ & $2.2 \pm 0.1$ & $25 \pm 02$ \\
\hline $\begin{array}{l}\text { W 2001/304/13:46:57 } \\
\text { [304.5743] }\end{array}$ & $\ldots$ & $\cdots$ & $\cdots$ & $\cdots$ & $\cdots$ & $3.0 \pm 0.4$ & $1.5 \pm 0.2$ & $415 \pm 23$ & $2.1 \pm 0.3$ & $14 \pm 17$ & $3.1 \pm 0.2$ & $1.7 \pm 0.1$ & $417 \pm 09$ & $2.4 \pm 0.1$ & $12 \pm 02$ \\
\hline $\begin{array}{l}\text { A } 2003 / 308 / 05: 59: 32 \\
\quad[308.2497]\end{array}$ & $3.1 \pm 1.1$ & $2.4 \pm 0.6$ & $779 \pm 34$ & $4.4 \pm 0.7$ & $43 \pm 4$ & $3.8 \pm 1.0$ & $2.8 \pm 0.6$ & $491 \pm 695$ & $2.7 \pm 2.8$ & $22 \pm 59$ & $\cdots$ & $\ldots$ & $\cdots$ & $\cdots$ & $\cdots$ \\
\hline $\begin{array}{l}\text { W 2003/308/06:46:01 } \\
\text { [308.2819] }\end{array}$ & $\cdots$ & $\cdots$ & $\cdots$ & $\cdots$ & $\cdots$ & $\cdots$ & $\cdots$ & $\cdots$ & $\cdots$ & $\cdots$ & $7.9 \pm 2.2$ & $2.8 \pm 0.8$ & $762 \pm 18$ & $2.7 \pm 0.3$ & $64 \pm 12$ \\
\hline $\begin{array}{l}\text { A } 2001 / 118 / 04: 31: 59 \\
\quad[118.1889]\end{array}$ & $3.7 \pm 0.8$ & $3.7 \pm 0.7$ & $905 \pm 59$ & $5.9 \pm 1.0$ & $88 \pm 02$ & $2.2 \pm 0.4$ & $2.4 \pm 0.3$ & $992 \pm 91$ & $5.0 \pm 1.0$ & $86 \pm 14$ & $3.3 \pm 0.7$ & $3.0 \pm 2.4$ & $906 \pm 58$ & $4.6 \pm 0.4$ & $90 \pm 04$ \\
\hline $\begin{array}{l}\text { W 2001/118/05:00:15 } \\
\text { [118.2085] }\end{array}$ & $\cdots$ & $\cdots$ & $\cdots$ & $\cdots$ & $\cdots$ & $1.2 \pm 0.9$ & $1.5 \pm 0.9$ & $1679 \pm 4663$ & $12.6 \pm 48$ & $48 \pm 58$ & $3.1 \pm 1.4$ & $3.0 \pm 0.2$ & $933 \pm 174$ & $4.9 \pm 0.3$ & $52 \pm 08$ \\
\hline
\end{tabular}

\section{Notes.}

${ }^{a}$ www.ssg.sr.unh.edu/mag/ace/ACElists/obs_list.html.

b ipshocks.fi/ (Kilpua et al. 2015).

www.cfa.harvard.edu/shocks/ using the method RH08 (Koval \& Szabo 2008). 
around Earth, was immersed in the solar wind regime. We will use particle data measured by SWICS on board ACE (Gloeckler et al. 1998), the Electron, Proton, and Alpha Monitor (EPAM) on board ACE (Gold et al. 1998), and the Three-Dimensional Plasma and Energetic Particle Investigation (3DP) on board Wind (Lin et al. 1995).

$A C E /$ SWICS is a linear time-of-flight mass spectrometer with electrostatic deflection. Using a combination of electrostatic deflection, a linear time of flight, and an energy measurement, SWICS measures the mass, charge, and energy of entering ions. After a particle has passed the instrument collimator, it enters the electrostatic analyzer. The analyzer is stepped through 60 logarithmic steps from $\sim 100$ to $\sim 0.5 \mathrm{kV}$ every 12 minutes. $A C E /$ SWICS has a nearly stationary field of view (integrated over a spacecraft spin and energy scanning period of 12 minutes) pointing nearly radially and sampling a nearly constant section of the sky. The SWICS instrument on $A C E$ is the improved flight spare unit of the original SWICS that traveled on board the Ulysses spacecraft (Gloeckler et al. 1992). Details of the $A C E /$ SWICS field of view, sensitivity, ion species distinction, and energy range covered can be found in Gloeckler et al. (1998) and Berger (2008).

$A C E / E P A M$ was the flight spare instrument of the Heliosphere Instrument for Spectra, Composition, and Anisotropy at Low Energies (HI-SCALE) on board Ulysses (Lanzerotti et al. 1992) and is composed of five telescope apertures of three different types. The Low Energy Magnetic Spectrometers, LEMS120 and LEMS30, of EPAM are dedicated to monitoring low-energy (46 keV-4.8 MeV) ions separated into eight energy channels (Gold et al. 1998). The LEMS30 (LEMS120) points at $30^{\circ}\left(120^{\circ}\right)$ from the spacecraft spin axis. Therefore, the LEMS30 system looks nearly into the solar wind, and LEMS120 looks mostly in the anti-sunward direction. In general, LEMS30 observes a higher intensity than LEMS120, which looks away from the solar wind. The differences caused by the Compton-Getting effect are significant for detailed studies when high time resolution and angular distributions are required (e.g., Lario et al. 2004). Some troublesome solar X-ray influences in the LEMS30 detector are present at times. For channels P1 (46-67 keV) and P2 (67-115 keV) of LEMS30, but also for channels P3-P8 in several cases, the sectors on the sunward side of the rotation are clearly contaminated by solar $\mathrm{X}$-rays and omitted when computing spin averages (hiscale. ftecs.com). Contamination by high-energy particles penetrating the instrument, occurring mainly at the onset of SEP events, might also contaminate the low-energy channels of both LEMS30 and LEMS120 (Marhavilas et al. 2015). Besides, the LEMS30 P1 channel has no data since day 327 of 2001, and P2 has no data since day 302 of 2003 (Haggerty et al. 2006). Gloeckler et al. (1995) combined Ulysses/SWICS with Ulysses/HI-SCALE data to construct proton energy spectra from thermal solar wind energies up to $\sim 5 \mathrm{MeV}$. These authors showed that HI-SCALE responded mostly to protons and that no ad hoc adjustment was required to bring the SWICS and HISCALE intensities into agreement.

Wind/3DP consists of six different detector arrays. In particular, we will use data from the ion electrostatic analyzer (PESA) that measures ions at 15 different energies from as low as $80 \mathrm{eV}$ to as high as $30 \mathrm{keV}$ (typical range in the solar wind is $500 \mathrm{eV}$ to $28 \mathrm{keV}$ ). This electrostatic analyzer, called
PESA-High (PESA-H), is mounted on a small boom, allowing for an almost unobstructed field of view of the $4 \pi$ sky and hence allowing us to generate pitch-angle distributions (e.g., Wilson et al. 2010). The detector has a relatively high threshold at low energies, making it difficult to detect background ions below $500 \mathrm{~km} \mathrm{~s}^{-1}(E \lesssim 1.5 \mathrm{keV})$ unless there is a well-defined peak. In other words, below $500 \mathrm{~km} \mathrm{~s}^{-1}$, the one-count level of the detector, which scales as $E^{-2}$, are too high to observe the particles often found in this energy range, which makes the observation of the solar wind thermal core difficult. Reflected UV light contributes also to producing a background instrumental intensity. At high speeds, PESA-H may also respond to penetrating higher-energy particles that contribute, in the most intense events, to the background intensity observed well before the arrival of shocks. During the passage of shocks associated with intense ESP components, PESA-H registers intensity increases above these background intensities that correspond to ions of the expected energy (i.e., $\sim 3$ to $\sim 28 \mathrm{keV})$. Wind/3DP high-energy $(>30 \mathrm{keV})$ ion data come from the Open Solid State Telescope (SST-O) that measures ions with energies up to $6 \mathrm{MeV}$ (Lin et al. 1995). The SST-O uses a common broom magnet to sweep away electrons below $\sim 400 \mathrm{keV}$ while leaving the protons relatively unaffected (Lin et al. 1995). Thus, in the absence of any higher-energy (penetrating) particles, the SST-O counts only ions. Neither Wind/3DP/PESA-H nor Wind/3DP/SST-O distinguish among protons, alphas, and other ion species.

In order to identify the shock passages and plasma properties upstream and downstream of the shocks, we use magnetic field data from the Magnetic Field Experiment (MAG) on ACE (Smith et al. 1998) and Magnetic Field Investigation (MFI; Lepping et al. 1995) on Wind, together with data from SWEPAM on ACE (McComas et al. 1998) and the Solar Wind Experiment (SWE) on Wind (Ogilvie et al. 1995).

We have selected five IP shocks of different characteristics that illustrate the different evolution that the ST proton population exhibits around shocks. Table 1 lists the main shock parameters of the shocks selected in this study. We specify the time of the shock passage by $A C E$ (A) and Wind (W) identified using magnetic field data. Shock parameters are obtained from the list of disturbances and transients observed by $A C E$, available at www.ssg.sr.unh. edu/mag/ace/ACElists/obs_list.html; the Database of Heliospheric Shock Waves generated by the University of Helsinki at ipshocks.fi (Kilpua et al. 2015); and the Harvard-Smithsonian Center for Astrophysics (CfA) Interplanetary Shock Database at www.cfa.harvard.edu/shocks/ using the method RH08 described in Szabo (1994) and Koval \& Szabo (2008). In particular, we list the density compression ratio $r_{n}$, the magnetic field compression ratio $r_{b}$, the shock speed in the spacecraft frame of reference $V_{s}$, the Alfvénic Mach number $M_{\mathrm{A}}$, the fast magnetosonic Mach number $M_{\mathrm{ms}}$, and the angle between the normal to the shock and the upstream magnetic field $\theta_{B n}$. The disparity of criteria applied to choose the regions representative of the upstream and downstream media and of the precise method used to compute the shock parameters may lead to different results. Additionally, Szabo et al. (2001) analyzed IP shocks observed by ACE, Wind, and $I M P-8$ and found that with large interspacecraft separation, large angular deviation between individual shock normals was possible, implying different shock parameters from one spacecraft to another. Therefore, it is not surprising that the same method 
applied to the shock observed at two different locations or the same shock using two different methods provides different shock parameters.

\subsection{Events with Upstream ST Proton Population Increasing First at High Energies}

\subsubsection{The Shock on Day 2001/023}

The left panels of Figure 1 show, as a function of time, (a) $A C E / \mathrm{SWICS}$ proton data in its 60 energy channels expressed as function of $1 / v$, where $v$ is the proton speed $\left(1 / v=0.5 \mathrm{~s} \mathrm{Mm}^{-1}\right.$ corresponds to $E \sim 20 \mathrm{keV}, 1 / v=1.0 \mathrm{~s}$ $\mathrm{Mm}^{-1}$ to $E \sim 5 \mathrm{keV}$, and $1 / v=1.5 \mathrm{~s} \mathrm{Mm}^{-1}$ to $E \sim 2.3 \mathrm{keV}$ ); (b) differential proton intensities for three $A C E / \mathrm{SWICS}$ channels artificially generated by adding counts over the indicated energy ranges and assuming isotropic distributions (orange, red, and black lines) and spin-averaged differential ion intensities measured in three energy channels of $A C E / E P A M /$ LEMS120 (blue and purple lines); (c)-(e) solar wind proton speed, density, and temperature measured by $A C E /$ SWEPAM; (f)-(h) magnetic field magnitude, elevation, and azimuth angle in the radial-tangential-normal (RTN) coordinate system as measured by $A C E / \mathrm{MAG}$; and (i) the angle ALPHA, defined as the angle between the radial direction and the magnetic field vector. The vertical solid line identifies the passage of the shock by the $A C E$ spacecraft. Figure 1(a) shows that prior to the passage of the shock (starting on day $~ 23.2$ ), ACE/SWICS intensities started to increase at the highest energies it can detect (i.e., $E \sim 80 \mathrm{keV}$ ). As the shock approached, the intensity increase was observed at lower energies, but just up to $1 / v=1.1 \mathrm{~s} \mathrm{Mm}^{-1}$ or $E \sim 4 \mathrm{keV}$ at the time of the shock passage. We have indicated in Figure 1(a) with a diagonal white dashed line the proton intensity increase observed by $A C E /$ SWICS prior to the shock arrival, suggesting that particles arrived at $A C E$ with signatures of velocity dispersion. At higher energies $(>50 \mathrm{keV}), A C E /$ EPAM/LEMS120 ion intensities gradually increased as the shock approached, reaching a peak just after the shock passage.

Figure 1(i) shows that starting on day $\sim 23.0$, the angle ALPHA oscillated around $\sim 90^{\circ}$ until day $\sim 23.15$, when it acquired values around $\sim 135^{\circ}$; then, on day $\sim 23.35$ (still upstream of the shock), it reached values close to $180^{\circ}$. If the ST protons observed prior to the shock passage are particles flowing along magnetic field lines, the measurement of these particles by $A C E /$ SWICS is favored when the magnetic field is directed along the field of view of the instrument, i.e., when the field is close to the radial direction. Therefore, the favorable field configuration for $A C E / \mathrm{SWICS}$ to observe ST particles moving along the field is when the angle ALPHA is close to either $0^{\circ}$ or $180^{\circ}$, whereas perpendicular configurations (ALPHA $\sim 90^{\circ}$ ) are unfavorable to detect particles streaming along the magnetic field. Note that ALPHA reached values close to $\sim 180^{\circ}$ for a short period of time before day $\sim 23.0$ (i.e., $\gtrsim 10 \mathrm{hr}$ before the shock passage), but $A C E /$ SWICS did not detect any ST intensity increase. Presumably, the shock was too distant from the spacecraft, and the foreshock ST particle region was not so extended as to be observed by $A C E /$ SWICS above its sensitivity level. One might argue that $A C E /$ SWICS observed only upstream ST protons close to the shock when ALPHA acquired values close to either $0^{\circ}$ or $180^{\circ}$. For this reason, we add Wind/3DP/PESA-H observations that allow a scan of the sky broader than that done by the stationary field of view of $A C E /$ SWICS. The disadvantage is the PESA-H does not distinguish among ion species and has an elevated instrumental background that hinders the measurements of weak signals.

The right panels of Figure 1 show, as a function of time, (j) spin-averaged ion differential intensities measured by Wind/ 3DP/PESA-H (top seven lines) and Wind/3DP/SST-O (bottom nine lines), (k)-(m) solar wind proton speed, density, and temperature measured by Wind/SWE, and (n)-(p) magnetic field magnitude, elevation, and azimuth angles in the geocentric solar ecliptic (GSE) coordinate system as measured by Wind/MFI. Figure 1(q) details the evolution of ST intensity pitch-angle anisotropies around the shock passage; in particular, it shows $10.5 \mathrm{keV}$ ion intensities measured by Wind/3DP/PESA-H transformed into the solar wind frame of reference binned in eight pitch angles (the values of the pitchangle cosine $\mu$ are color-coded and listed in the figure; reddish lines correspond to pitch-angle cosines $\mu \sim-1$, whereas greenish lines correspond to pitch-angle cosines $\mu \sim 1$ ). Similar to $A C E /$ SWICS, Wind/3DP/PESA-H observed a gradual increase starting around $\sim 23.2$ and only at energies $\gtrsim 5 \mathrm{keV}$, whereas SST-O exhibited a gradual increase for energies $\lesssim 800 \mathrm{keV}$ peaking just downstream of the shock. Figure 1(q) shows weak anisotropy, where particles with $\mu \sim-1$ dominated over those with $\mu \sim 0$ or 1 (considering the upstream magnetic field polarity $\phi_{\mathrm{GSE}} \sim 0^{\circ}$, negative pitchangle cosines indicate particles moving anti-sunward). Therefore, the particle enhancement just before the shock arrival was preponderant in the field-aligned direction, although it was observed for all pitch angles.

The top row of Figure 2 shows the proton energy spectra observed by $A C E /$ SWICS (orange and red dots) and the spinaveraged ion energy spectra as observed by $A C E / E P A M /$ LEMS120 (blue dots) and ACE/EPAM/LEMS30 (gray dots) at different times around the passage of the shock. The orange and red dots distinguish those $A C E /$ SWICS data points in the thermal and ST regimes, respectively. The green dashed line indicates the one-count level of $A C E / \mathrm{SWICS}$ in the ST regime. The differential fluxes from $A C E$ /SWICS plotted in Figure 2 have been computed assuming isotropic proton distributions. Each panel contains a 12 minute period in which $A C E /$ SWICS scanned all energies sampled by this instrument. The label in each panel indicates the initial time (in units of fractional day) of each one of these 12 minute intervals (ACE/EPAM data have been averaged over this 12 minute interval). At the bottom of each panel, we indicate the time in minutes remaining for the passage of the shock (negative values) or lagging the shock passage (positive values). We should indicate that $A C E /$ SWICS was not designated to measure the thermal component of the solar wind protons, and therefore there can be some differences between this component of the spectra and the solar wind parameters obtained from $A C E / S W E P A M$ measurements. The top row of Figure 2 shows that the ST portion of the energy spectrum (red dots) was gradually populated as the shock approached, starting at high energies and exhibiting a bump peaking around $\sim 10 \mathrm{keV}$ just before the arrival of the shock (indicated by the black arrow in Figure 2(e)). Figure 2(f) covers a 12 minute interval that starts upstream but spans a large fraction of the downstream region, whereas Figure 2(g) completely spans the downstream region. The smooth continuity of the spectra between $A C E /$ SWICS and $A C E /$ EPAM measurements in the downstream region indicates the 


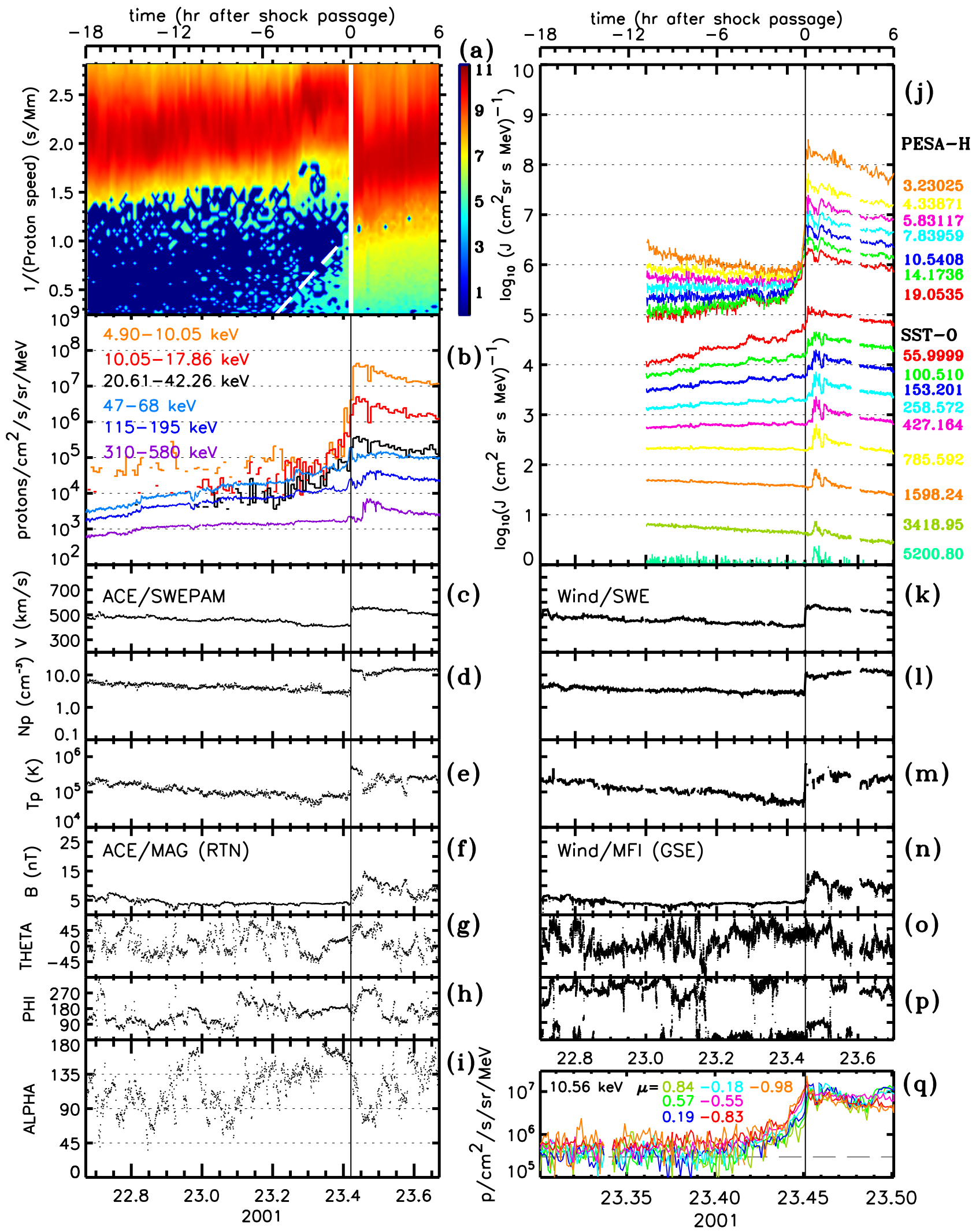

Figure 1. (a) $A C E /$ SWICS proton data as a function of $1 / v$, where $v$ is the particle velocity. (b) Proton intensities as measured by SWICS in artificial generated channels (red, orange, and black lines) and 12 minute averaged ion intensities measured by ACE/EPAM/LEMS120 (blue and purple lines). The ACE/SWICS proton differential fluxes have been computed assuming isotropic intensities. Solar wind proton (c) speed, (d) density, and (e) temperature measured by ACE/SWEPAM. Magnetic field (f) magnitude, (g) elevation, and (h) azimuth angles in the spacecraft-centered RTN coordinate system measured by ACE/MAG. (i) Angle ALPHA between the magnetic field vector and the radial direction. (j) Ion intensities measured by Wind/3DP/PESA-H (top lines) and Wind/3DP/SST-O (bottom lines). Solar wind proton (k) speed, (l) density, and (m) temperature measured by Wind/SWE. Magnetic field (n) magnitude, (o) elevation, and (p) azimuth angles in the GSE coordinate system measured by Wind/MFI. (q) The $\sim 10.5 \mathrm{keV}$ ion intensities for different pitch-angle cosines $(\mu)$ as measured by Wind/3DP/PESA-H. The vertical lines indicate the passage of the IP shock. 

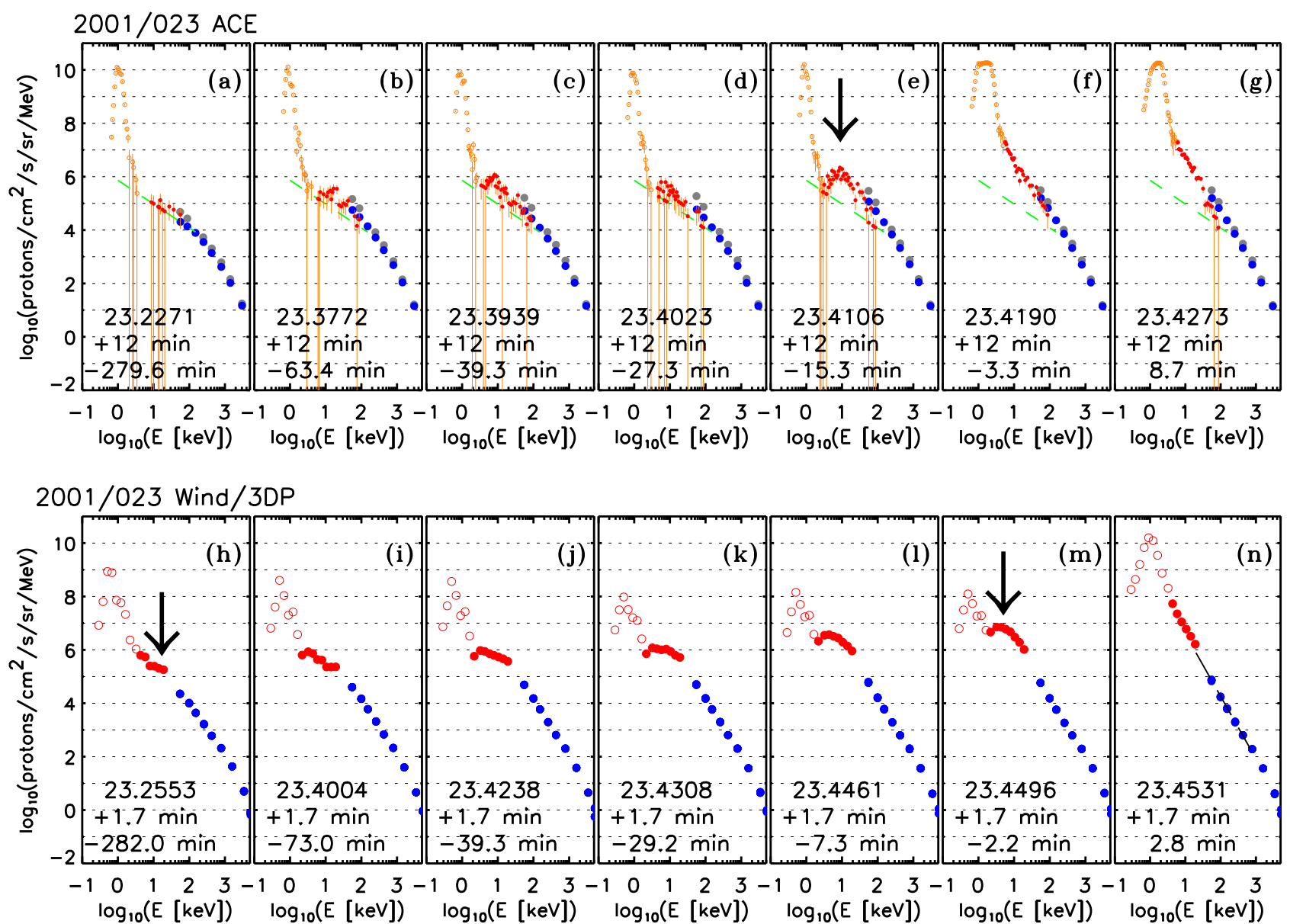

Figure 2. (a)-(g) Energy spectra of proton intensities in the spacecraft frame of reference as measured by ACE/SWICS (orange and red dots) and of spin-averaged ion intensities measured by ACE/EPAM/LEMS120 (blue dots) and ACE/EPAM/LEMS30 (gray dots) at different times before and after the passage of the shock. The time in each panel (in units of fractional day of the year) indicates the initial time of the 12 minutes over which ACE/SWICS data have been collected and ACE/ EPAM averaged. The dashed green lines indicate the one-count level of $A C E /$ SWICS in the ST energy regime. The thin orange lines indicate the error bar associated with each point based on Poisson statistics. (h)-(n) Energy spectra of the spin-averaged ion intensities in the spacecraft frame of reference as measured by Wind/3DP/ PESA-H (red dots) and Wind/3DP/SST-O (blue dots) at different times before and after the passage of the shock. The time in each panel (in units of fractional day of the year) indicates the initial time of the 1 minute $40 \mathrm{~s}$ over which Wind/3DP data have been averaged. The time indicated at the bottom of each panel (in units of minutes) is the time before (negative values) or after (positive values) the shock passage at each respective spacecraft.

agreement between intensities measured by both instruments, at least when particle distributions are isotropic, as expected in the far downstream region of shocks. In the energy range $40-500 \mathrm{keV}$, the downstream energy spectrum approaches a power law $E^{-\gamma}$ with index $\gamma=2.14 \pm 0.23$.

The bottom row of Figure 2 shows the spin-averaged ion energy spectra observed by Wind/3DP/PESA-H (red dots) and Wind/3DP/SST-O (blue dots) in the spacecraft frame of reference. Similar to ACE/SWICS, PESA-H was not designed to measure the thermal portion of the solar wind spectra, and we have indicated this portion with red open circles. Each panel covers $100 \mathrm{~s}$ of data starting with the time indicated in each panel (in units of fractional day). Similar to the ACE/SWICS energy spectra, the intensities started increasing above background at the highest energies of PESA-H $(\sim 20 \mathrm{keV}$, indicated by the black arrow in Figure 2(h)). As the shock approached, the intensity enhancement gradually moved to lower energies, reaching a peak at $\sim 6 \mathrm{keV}$ just before the arrival of the shock (indicated by the black arrow in Figure 2(m)). Downstream of the shock (Figure 2(n)), the spectra smoothly concatenate PESA-H and SST-O intensities. Between 40 and $500 \mathrm{keV}$, a power law $E^{-\gamma}$ with slope $\gamma=2.33 \pm 0.20$ was a good representation for the energy spectra downstream of the shock and consistent with the index measured at $A C E$.

Despite the different locations of Wind and $A C E$ and the different properties of the shocks (Table 1), the ST particle signatures were similar at both spacecraft. Both spacecraft detected an upstream ST intensity enhancement with velocity dispersion signatures and relatively weak anisotropies. The ST energy spectra populated first at high energies, and as the shock approached, a bump developed that, immediately before the shock passage, peaked at $\sim 6-10 \mathrm{keV}$.

\subsubsection{The Shock on Day 2002/198}

The left panels of Figure 3 show, with the same format as Figure 1, ACE data for the intense SEP event on day 198 of 2002. ACE/EPAM intensities kept increasing long before the arrival of the shock (as part of the prompt component of the SEP event) and peaked coinciding with the shock passage. In contrast, $A C E$ /SWICS intensities only increased $\sim 1 \mathrm{hr}$ before the shock arrival, starting first at the highest energies of $A C E$ / SWICS $(\sim 50 \mathrm{keV})$. Low-energy proton intensities increased only just before the shock arrival. Such an increase was significant only at energies $\gtrsim 5 \mathrm{keV}$. Throughout the upstream 


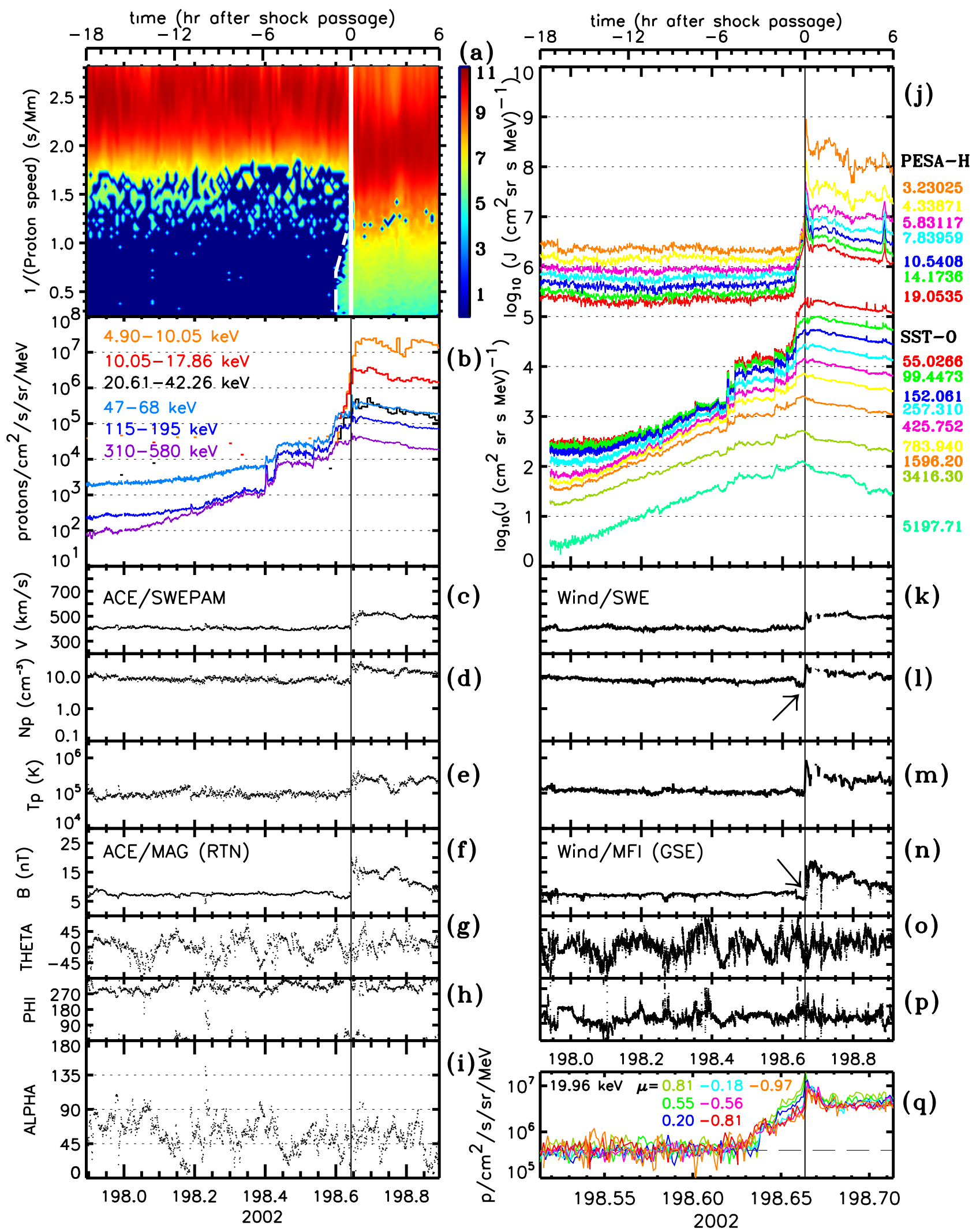

Figure 3. Same as Figure 1 but for the shock on day 198 of 2002.

region, the angle ALPHA oscillated around $\sim 45^{\circ}$, which may hinder the detectability of ST particles by $A C E /$ SWICS. The upstream ST proton enhancement did not coincide with a sudden change of ALPHA.
The right panels of Figure 3 show, with the same format as Figure 1, Wind data that exhibit very similar characteristics as those from $A C E$. The PESA-H intensity enhancement started at $\sim 20 \mathrm{keV}$ just $\sim 1 \mathrm{hr}$ before the shock arrival, coinciding also 

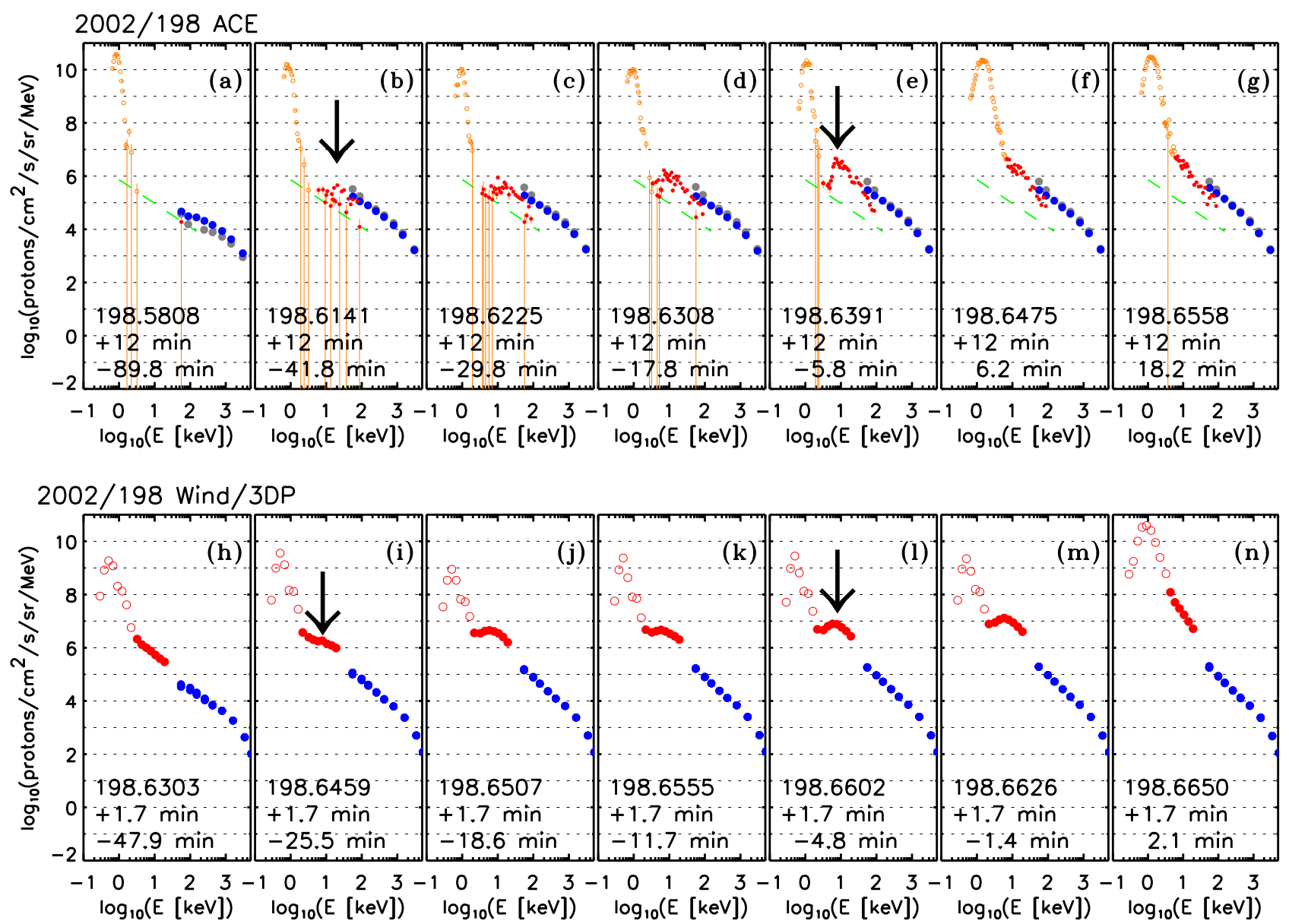

Figure 4. Same as Figure 2 but for the shock on day 198 of 2002.

with the final upstream increase observed by SST-O at energies below 1.5 MeV. We emphasize that the increase of ST particles immediately before the shock coincided with a decrease of solar wind density and magnetic field magnitude (indicated by arrows in Figures 3(l) and (n)). Particle intensity enhancements concurrent with solar wind density and magnetic field depressions have previously been observed in ESP events where there is a balance between the sum of the thermal and magnetic field pressures and the pressure exerted by energetic particles (Lario et al. 2015). Figure 3(q) shows that the final upstream increase of $\sim 20 \mathrm{keV}$ ions was relatively anisotropic. Note that the polarity of the magnetic field was outward along the nominal Parker spiral direction $\left(\phi_{\mathrm{GSE}}=135^{\circ}\right)$; hence, the dominant intensities were observed at pitch-angle cosines $\mu \sim 1$, as indicated by the greenish lines in Figure 3(q) (i.e., particles flowing in the anti-sunward direction along the magnetic field).

Figure 4 shows, with the same format as Figure 2, the evolution of the energy spectra around the shock passage. Similar to the event shown in Figure 2, the ST population starts increasing at high energies (black arrow in Figures 2(b) and (i)) but now starting just $\sim 45$ minutes before the shock passage. As the shock approached, the energy spectra gradually evolved to a bump with a peak at $\sim 10 \mathrm{keV}$ just before the shock passage (indicated by the black arrows in Figure 4(e) and (1)). The energy spectrum only adopts a power-law dependence at later times in the downstream region. In the energy range $40-500 \mathrm{keV}$, a least-squares fit provides a dependence $E^{-1.07 \pm 0.18}$ in Figure $4(\mathrm{~g})$ and $E^{-1.31 \pm 0.01}$ in Figure 4(n).

In many aspects, the event on $2002 / 198$ is similar to the event on 2001/023 (Section 2.1.1), in terms of the weakly anisotropic ST intensity increase observed upstream of the shock and the development of a bump in the energy spectra immediately before the shock arrival. However, the ST intensity enhancement prior to the shock passage was much more limited in time during the 2002/198 event than during the 2001/023 event, especially at energies $\sim 10-20 \mathrm{keV}$. The disparity of parameters inferred for these two shocks in the different catalogs (Table 1) does not allow us to discriminate whether the differences between the two events are due to a change of shock obliquity, as one should expect if the shock on 2001/023 was more parallel than the shock on $2002 / 198$, allowing for an easy escape of particles streaming along the field lines in the upstream region. On the contrary, we believe that the upstream cavity observed immediately upstream of the shock on 2002/198 (with depressed solar wind density and magnetic field magnitude) played a role in the limited extension of the upstream ST intensity enhancement by confining low-energy ions (see similar examples in Lario et al. 2015, and references therein).

\subsection{Events with Upstream ST Proton Population Increasing Only at Low Energies: The Shock on Day 2001/304}

The left panels of Figure 5 show, with the same format as Figure 1, ACE data for the shock on 2001/304. High-energy 


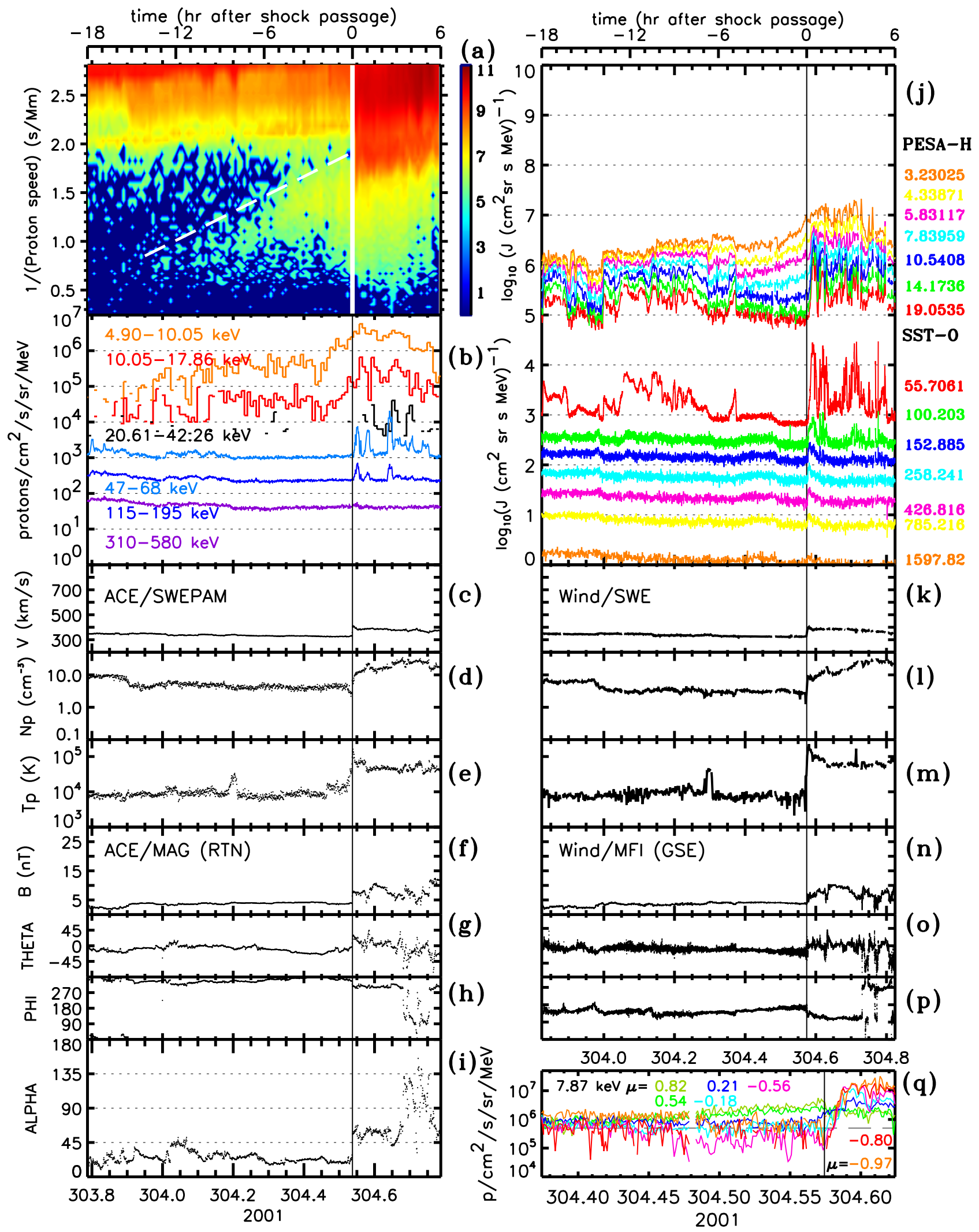

Figure 5. Same as Figure 1 but for the shock on day 304 of 2001.

( $\gtrsim 50 \mathrm{keV}$ ) particles only exhibited intensity enhancements downstream of the shock. The upstream region for this shock was characterized by low solar wind proton temperatures $\left(\sim 10^{4} \mathrm{~K}\right.$ or $\sim 0.86 \mathrm{eV})$, relatively slow solar wind $\left(\sim 320 \mathrm{~km} \mathrm{~s}^{-1}\right)$, and a smooth magnetic field. The shock was running into a structure categorized as the IP counterpart of a coronal mass ejection (ICME) by I. G. Richardson and H. V. Cane (www.srl.caltech.edu/ACE/ ASC/DATA/level3/icmetable2.htm). Figure 5(a) shows that, prior 

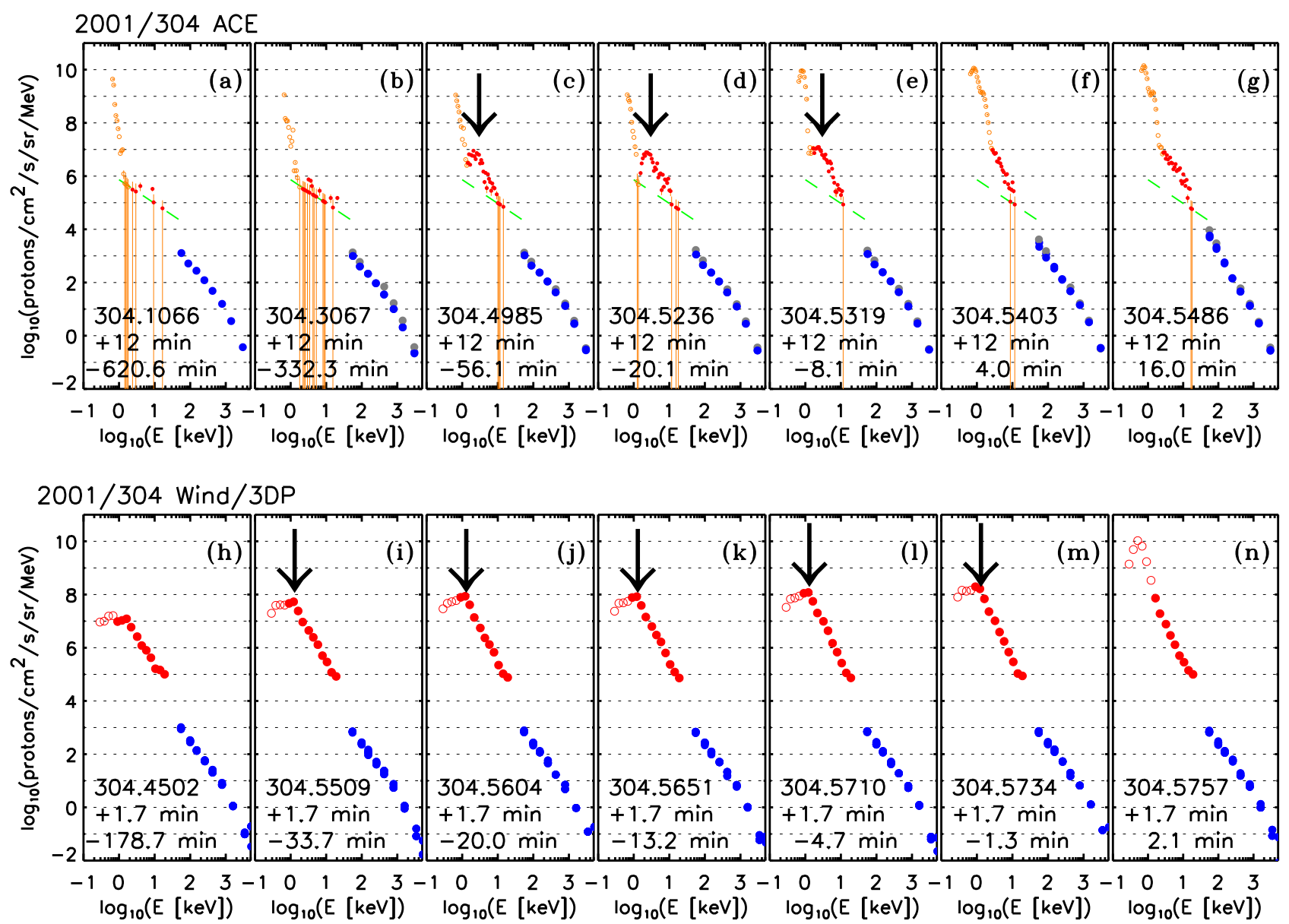

Figure 6. Same as Figure 2 but for the shock on day 304 of 2001.

to the passage of the shock (at 304.0), ACE/SWICS intensities started to increase first at $1 / v \sim 0.7 \mathrm{~s} \mathrm{Mm}^{-1}$ $(E \sim 10 \mathrm{keV})$. As the shock approached, the intensity increase was observed at lower and lower energies (indicated by the white dashed line in Figure 5(a)), drastically intensifying at energies below $\sim 5 \mathrm{keV}$ at around $\sim 304.3$. At higher energies $(>50 \mathrm{keV})$, ion intensities remained flat through the upstream region (Figure 5(b)). Upstream of the shock, the magnetic field was close to the radial direction, with an anti-sunward orientation (Figure 5(h)) implying a small angle ALPHA (Figure 5(i)) and therefore a good configuration for $A C E$ / SWICS to detect particles if those were flowing along the magnetic field. The radial upstream magnetic field and the nearly radial shock-normal orientations indicate a parallel shock (i.e., virtually no $V \times B$ electric field) that, together with the low upstream solar wind temperature (with a less energetic upstream population), implies an inefficient shock in the acceleration of particles to high energies and hence that the ESP event was observed only at energies $\lesssim 10 \mathrm{keV}$.

The right panels of Figure 5 show Wind data for the shock on $2001 / 304$. With the exception of the $\sim 55 \mathrm{keV}$ channel of SST$\mathrm{O}$, the Wind measurements are very similar to those by $A C E$. Particle enhancements prior to the shock arrival were observed only at low energies, with enhancements immediately upstream of the shock at low $\lesssim 10 \mathrm{keV}$ ion energies. Figure 5(q) shows that the $\sim 8 \mathrm{keV}$ ion intensity increased immediately before the shock was highly anisotropic, with only $\mu \sim 1$ intensities (greenish traces) increasing for $\sim 4 \mathrm{hr}$ before the shock arrival (the radially outward magnetic field orientation with $\phi_{\mathrm{GSE}} \sim 180^{\circ}$ indicates that particles with $\mu \sim 1$ propagated in the anti-sunward direction). It has been suggested that the smooth magnetic field characteristic of ICMEs provides the appropriate conditions for the scatter-free propagation of particles (e.g., Torsti et al. 2004). Moreover, the catalogs of shock parameters agree with this shock being quasi-parallel. Therefore, the conditions were propitious for the observation of field-aligned particles streaming out of the shock.

Figure 6 shows, with the same format as Figure 2, the evolution of the energy spectra around the shock on 2001/304. The low solar wind speed and temperature upstream of the shock prevented a reliable measurement of the thermal portion of the energy spectra by both $A C E /$ SWICS and PESA-H. The $A C E /$ SWICS data show that the ST energy regime started to populate only at low energies $(\lesssim 10 \mathrm{keV})$. As the shock approached, the energy spectra developed in a profile with a hump peaking at $\sim 3 \mathrm{keV}$ (indicated by the black arrows in Figures 6(c)-(e)). The hump in the energy spectra built using PESA-H measurements is not so well developed due to the difficulty of this instrument close to thermal energies, but we can discern a peak at around $\sim 1.5 \mathrm{keV}$, indicated by the black arrows in Figures 6(i)-(m). 




Figure 7. Same as Figure 1 but for the shock on day 308 of 2003. 

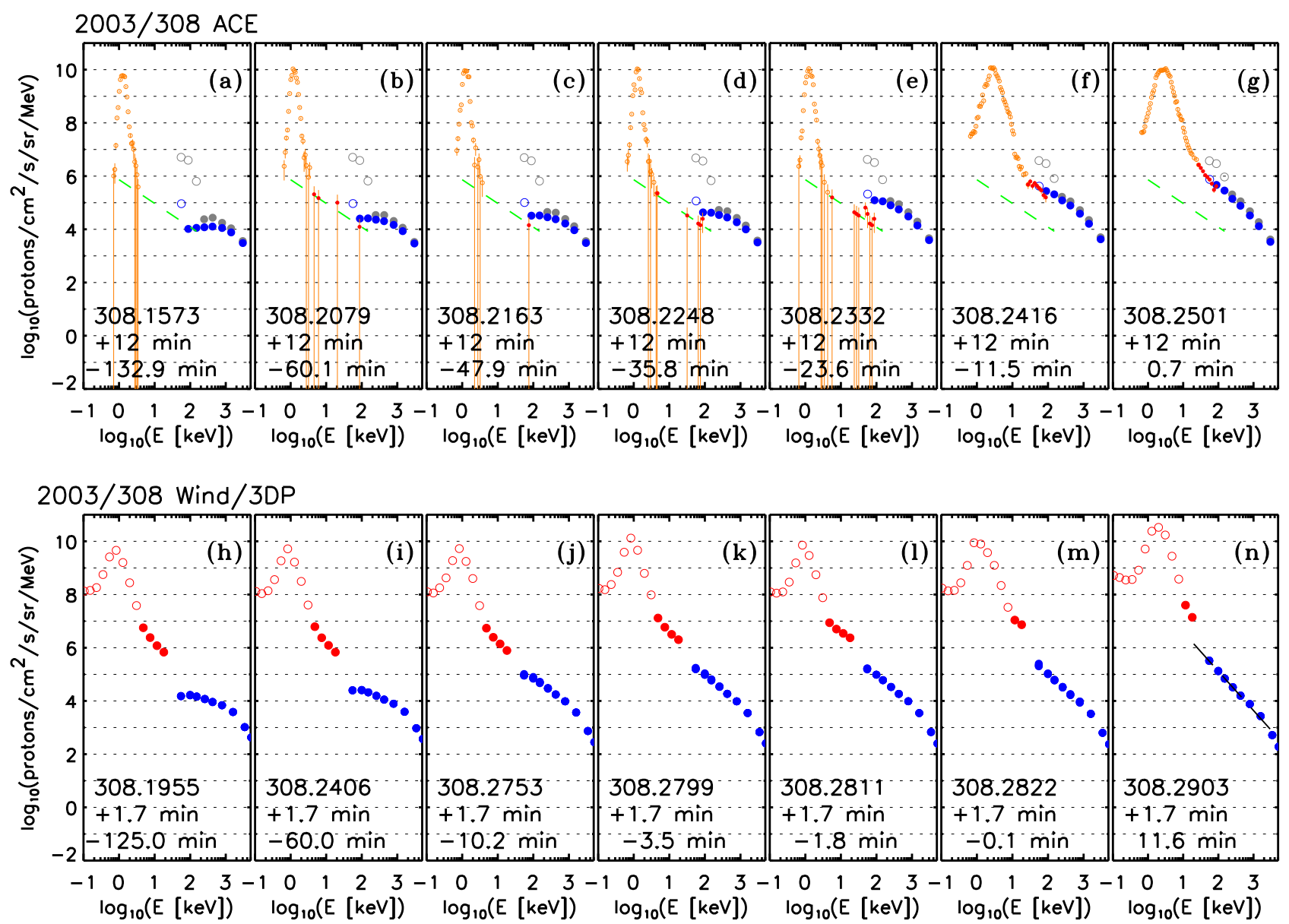

Figure 8. Same as Figure 2 but for the shock on day 308 of 2003.

\subsection{Events without Measured ST Population Upstream of the Shock}

\subsubsection{The Shock on Day 2003/308}

Figure 7 shows, with the same format as Figure 1, ACE and Wind data for the shock observed on 2003/308. This ESP event was associated with one of the intense SEP events in the series of events of 2003 October-November (see Figure 4 in Lario et al. 2005a). Figure 7(a) shows the absence of ST particles measured by $A C E /$ SWICS throughout the upstream region of the shock. Figure 7(b) shows that, apart from discrete counts in the artificially generated $4.9-10 \mathrm{keV}$ proton channel (orange line), $A C E$ /SWICS did not detect any proton increase upstream of the shock. The only exception is the last 12 minute interval prior to the shock arrival in the highest energies of $A C E$ / SWICS (black line in Figure 7(b)). The energy channel $47-68 \mathrm{keV}$ of $A C E /$ EPAM/LEMS120 was contaminated by penetrating high-energy particles, whereas the higher-energy channels of $A C E /$ EPAM/LEMS120 gradually increased with the arrival of the shock (blue and purple lines in Figure 7(b)). The angle ALPHA oscillated around $\sim 135^{\circ}$, which might hinder the observation of ST protons by ACE/SWICS if those particles were present and flowing along the magnetic field.

Wind/3DP/SST-O data exhibited periods of flat spectra throughout the upstream region of this shock. These periods are easily identifiable in Figure 7(j), as those time intervals when intensities of different energies (between $55 \mathrm{keV}$ and $1.5 \mathrm{MeV}$ ) overlap. As the shock approached, the intensities in the different channels of SST-O separated, resulting in a steepening of the energy spectra. By contrast, Wind/3DP/PESA-H exhibited elevated intensities several hours prior to the shock arrival that gradually decreased with time. The elevated intensities of PESA$\mathrm{H}$ resulted from penetrating high-energy particles of the prompt component of the SEP event already on day 306 (not shown here). Just about $1 \mathrm{hr}$ before the arrival of the shock, PESA-H intensities increased, which might constitute ST particles associated with the shock. Figure 7(q) shows that the anisotropy associated with this intensity increase was very weak with higher intensities at $\mu \sim-1$ (that according to the field polarity corresponds to particles moving in the anti-sunward direction, although all intensities at different pitch angles increased immediately before the shock arrival).

Figure 8 shows the evolution of the energy spectra around the passage of the shock on $2003 / 308$. In the top panels, we indicate with open symbols when the particle intensities in the lowest-energy channels of ACE/EPAM/LEMS120 (blue) and $A C E / E P A M / L E M S 30$ (gray) departed from the spectra profile because of penetrating high-energy particles. The flattening of the high-energy component of the spectra raises the question of whether there is a connection between the thermal and highenergy components of the spectra. However, ACE/SWICS did not provide any counts above its sensitivity one-count level. The only ST upstream counts provided by ACE/SWICS were at the highest-energy end in the 12 minute interval prior to the shock arrival (Figure 8(e)). The bottom panels of Figure 8 show 
that the elevated intensities measured by PESA-H already upstream of the shock resulted in an ST tail exhibiting a powerlaw dependence that most likely was due to the decaying intensities observed well before the shock arrival (Figure 7(j)). This tail does not smoothly connect with the high-energy component of the spectra (blue symbols) that had flattening signatures (Figures 8(h)-(i)), which may be due to the presence of SEPs affecting the high-energy counts of PESA-H. The intensity in this ST tail was only enhanced as the shock approached (Figures 8(j)-(1)) but without exhibiting any bump or clear peak, as shown in the prior events. Immediately before the shock passage, the slope of the spectra changed (Figure $8(1))$ as the higher energies $(\sim 20 \mathrm{keV})$ increased more than the lower energies. Far in the downstream region, highenergy $(>50 \mathrm{keV})$ particles reached a power-law spectrum $E^{-\gamma}$ with $\gamma=1.47 \pm 0.09$ in the energy range $50-500 \mathrm{keV}$ (black dashed line in Figure 8(n)), but that does not smoothly connect with the PESA-H measurements affected by the large downstream plasma temperature (Figure $7(\mathrm{e})-(\mathrm{m})$ ).

\subsubsection{The Shock on Day 2001/118}

Figure 9 shows the intense ESP event on 2001/118. The main characteristic of this event is the nonobservation of ST protons by $A C E /$ SWICS throughout the upstream region. The angle ALPHA oscillated between $45^{\circ}$ and $90^{\circ}$ (Figure 9(i)), which might raise the question about $A C E / \mathrm{SWICS}$ detection of ST protons if those particles were present and flowing along the magnetic field. The Wind/3DP data shown in Figure 9(j) show that PESA-H intensities were at instrumental background level throughout the upstream region with the exception of just $\sim 2$ minutes before the shock passage. Figure $9(\mathrm{q})$ details the $\sim 14 \mathrm{keV}$ ion intensity increase observed in association with the shock passage. Note that intensities in Figure 9(q) are in the solar wind frame of reference. We use Wind/SWE data for this transformation. The Wind/SWE data gap at the time of the shock passage (Figures $9(\mathrm{k})-(\mathrm{m})$ ) prevents us from obtaining a pitch-angle distribution just at the time of the shock passage in Figure 9(q). The last data point prior to the shock passage indicates a weakly anisotropic enhancement with predominant intensities at $\mu \sim 1$ (greenish lines). At higher energies, both $A C E /$ EPAM and Wind/3DP/SST-O data showed similar ion intensities at energies $\lesssim 100 \mathrm{keV}$, indicating a flat energy spectrum (between $\sim 50$ and $\sim 150 \mathrm{keV}$ ) throughout the upstream region (Lario et al. 2018).

Figure 10 shows the evolution of the energy spectra around the shock passage. Throughout the upstream region, $A C E /$ SWICS did not observe significant counts in the ST regime (Figures 10(a)-(d)). It is only downstream when a continuous energy spectrum forms. PESA-H intensities did not start increasing until $\sim 7$ minutes prior to the shock passage (Figure 10(j)), and it is not until 2 minutes before the shock passage when a bump peaking at $\sim 8 \mathrm{keV}$ develops in the energy spectra (Figures 10(1)-(m)). The absence of a distinct bump in the $A C E /$ SWICS data might have resulted from either the time resolution used to scan the different energies with this instrument that led it to miss this limited particle increase or the fact that the perpendicular magnetic field (ALPHA $\sim 90^{\circ}$ ) did not favor the observation of this presumably anisotropic particle increase.

\section{Discussion and Summary}

The time evolution of the ST proton population around shocks depends on (1) the efficiency of both shocks and downstream plasma in accelerating particles (which depends on the properties of both the shocks and the downstream compression regions, as well as the presence and properties of the seed particles subject to being accelerated) and (2) the ability of the particles to propagate away from the shocks (which depends on the relative speed of the particles with respect to the shock velocity along the upstream magnetic field and also on the plasma conditions found upstream and downstream of the shocks). Our analysis focuses on item (2), since it has been proven that shock parameters do not unequivocally determine the properties of the ESP events (e.g., Lario et al. 2005b), the role of the compression regions has not been completely quantified (Fisk \& Gloeckler 2012; Zank et al. 2015), and the injection of particles from a seed population into the processes of particle acceleration depends not only on the shock parameters but also, among other factors, on the presence of magnetic field fluctuations upstream of the shocks (Giacalone 2005) and hence the diffusion processes undergone by the particles around the shocks (Zank et al. 2006). Additionally, other factors that may affect the evolution of the ST populations around shocks not addressed here include the microstructure of the shocks that differentiates them from planar structures on ion or electron scales and the fact that shocks are not laminar and not necessarily stationary but can reform.

Particle escape from the shock is determined by the conditions that control the particle transport around the shock. Under the assumption that particles propagate scatter-free along magnetic field lines and that these field lines are straight on the scale of the particle foreshock, the escape of particles from the shock vicinity occurs when their speed parallel to the magnetic field $v_{\|}$is larger than the speed of the shock along the magnetic field in the upstream flow frame. Therefore, particles departing from the shock front are able to reach distances farther away from the shock in parallel $\left(\theta_{B n} \sim 0^{\circ}\right)$ than in perpendicular $\left(\theta_{B n} \sim 90^{\circ}\right)$ configurations (e.g., Burgess 1995). When the upstream plasma flow in the shock frame of reference is directed along the shock normal $\hat{n}$, we can express the speed of the shock along the field line in the upstream flow frame of reference as $U_{\text {up }} \sec \theta_{B n}$, where $U_{\text {up }}$ is the upstream flow velocity in the shock frame (e.g., Burgess 1995). In this configuration, the observation of particles far upstream from a

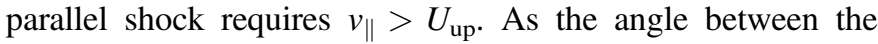
shock normal and upstream magnetic field becomes closer to perpendicular, it becomes increasingly difficult for a particle to escape from the shock. Additionally, particle gyration contributes to bringing upstream particles back to the shock, especially at perpendicular shocks. Therefore, particles with small pitch angles (i.e., $|\mu| \sim 1$ ) are able to propagate farther away from the shock than particles with $|\mu| \sim 0$. The energy and pitch-angle dependence of the escaping particles produces a velocity dispersion effect seen at the onset of the upstream ESP intensity enhancements. The inability of low-energy particles to escape from the shock results in the development of a hump in the energy spectra peaking at a certain energy that depends on the relative difference between $v_{\|}$and the shock speed along the upstream field lines (e.g., le Roux \& Webb 2012). The amplification of waves by particles streaming out of 


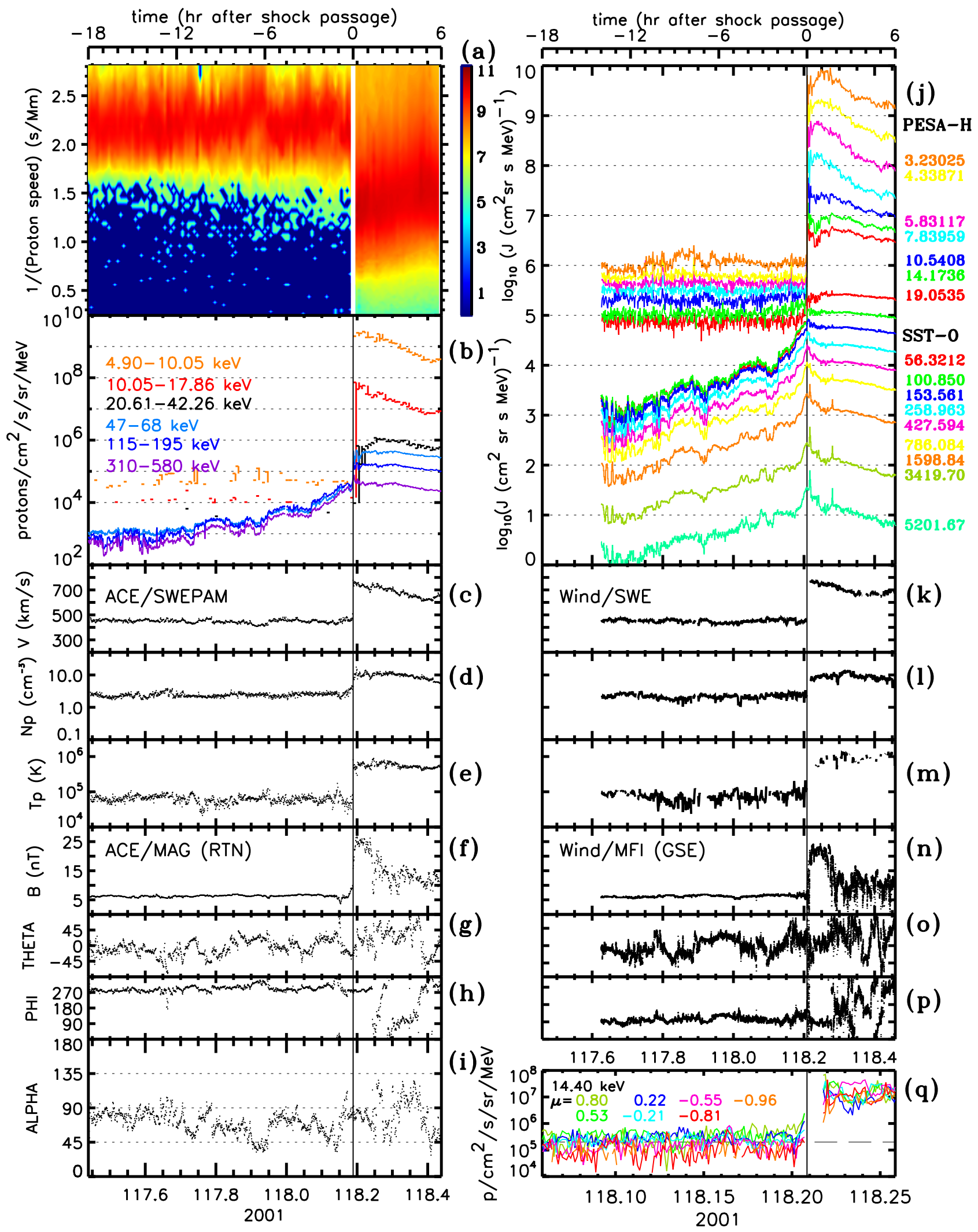

Figure 9. Same as Figure 1 but for the shock on day 118 of 2001.

the shock modifies the propagation conditions for the particles, resulting in more scattering processes that reduce $v_{\|}$and hence the capability of the particles to escape from the shock and the development of a hump in the energy spectra (e.g., Giacalone et al. 1993).
Typically, the upstream plasma flow $\boldsymbol{U}$ is not completely parallel to the shock-normal direction $\hat{n}$, but it may form an angle $\theta_{U n}$ with $\hat{n}$. The component of the upstream plasma velocity $U_{t}$ perpendicular to $\hat{n}$ and lying on the plane formed by the upstream magnetic field $\boldsymbol{B}$ and $\hat{n}$ contributes to the speed of 

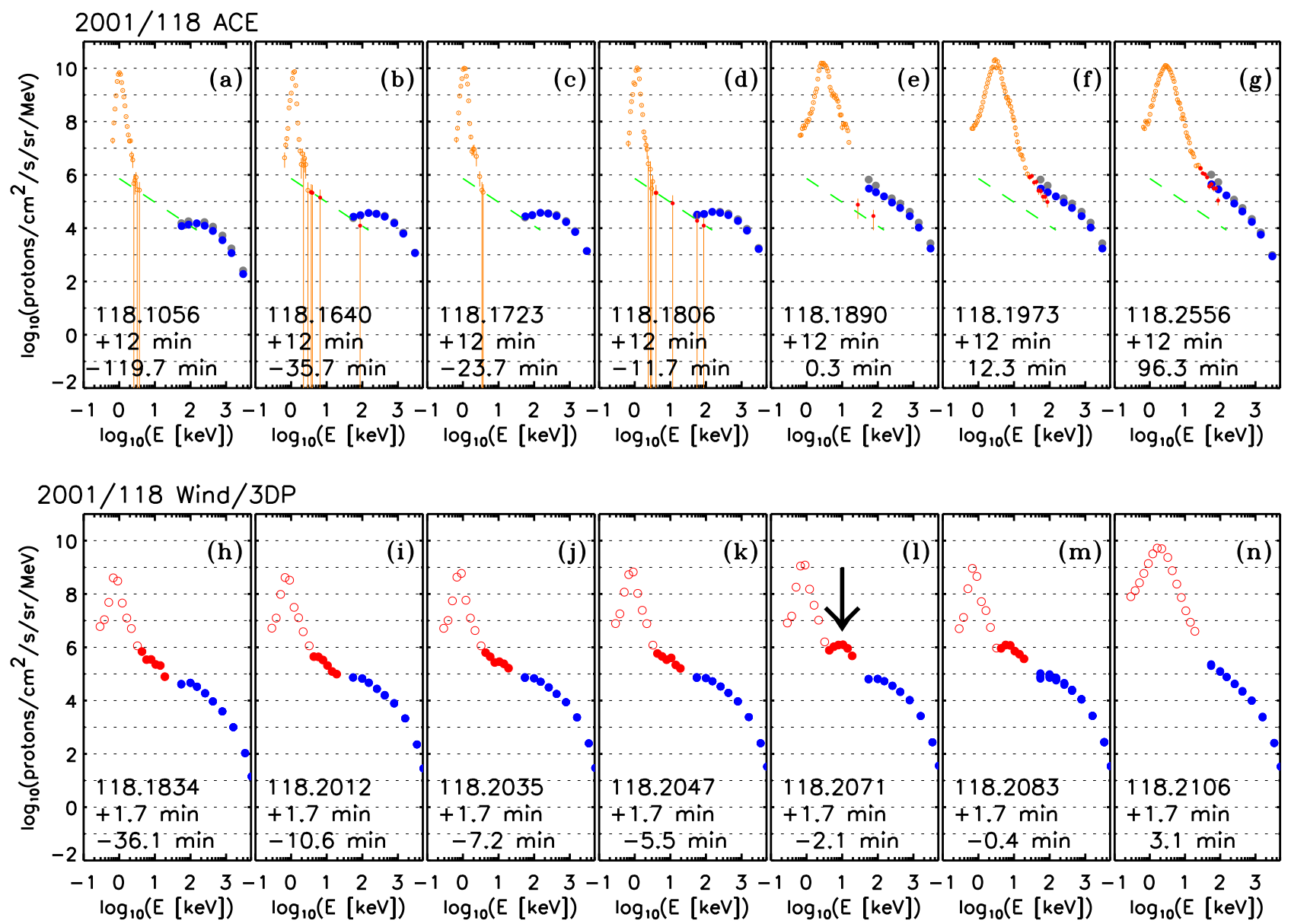

Figure 10. Same as Figure 2 but for the shock on day 118 of 2001.

the shock along the upstream magnetic field direction $W_{b}$ measured in the upstream frame of reference. The speed $W_{b}$ of the shock along $\boldsymbol{B}$ is just the hypotenuse of the right-angled triangle with one side $U_{n}$, which is normal to the shock surface, and the other side $U_{t}-U_{n}\left(B_{t} / B_{n}\right)$ (i.e., the transformation speed into the de Hoffmann-Teller frame; e.g., Gurnett \& Bhattacharjee 2005), which is along the shock surface, in which case $W_{b}$ can be expressed as

$$
W_{b}=\left[U_{n}^{2}+\left(U_{t}-U_{n} \frac{B_{t}}{B_{n}}\right)^{2}\right]^{1 / 2},
$$

where $U_{n}$ and $B_{n}$ are the components of the upstream flow and upstream magnetic field along $\hat{n}$, and $U_{t}$ and $B_{t}$ are the components of the upstream flow and upstream magnetic field perpendicular to $\hat{n}$ and lying on the plane formed by $\boldsymbol{B}$ and $\hat{n}$ (i.e., $B_{n}=B \cos \theta_{B n}$ and $B_{t}=B \sin \theta_{B n}$ ). Note that when $U_{t}=0$, the upstream flow is along the normal; i.e., $U_{n}=U_{\text {up }}$, and therefore $W_{b}$ simplifies to $U_{\text {up }} \sec \theta_{B n}$. For each selected event, Table 2 lists the shock speed in the spacecraft frame of reference $V_{\text {sh }}$ along the normal direction, the upstream solar wind velocity $\boldsymbol{V}_{\mathrm{sw}}$ in the spacecraft frame of reference, the upstream magnetic field $\boldsymbol{B}$, and the normal vector $\hat{n}$ obtained from the ACE Transient List (columns 2-5) and the ipshocks.fi catalog (columns 7-10). We have used these parameters to compute $\theta_{U n}$ (listed in columns 6 and 11), as well as $W_{b}$ and $v_{\text {esc }}=U_{\text {up }} \sec \theta_{B n}$, where $U_{\text {up }}$ has been computed as $U_{\text {up }}=$ $\left|V_{\mathrm{sh}}-\boldsymbol{V}_{\mathrm{sw}} \cdot \hat{n}\right|$ and $\theta_{B n}$ from the vectors $\boldsymbol{B}$ and $\hat{n}$ listed in Table 2. Note that for the shock on 2001/023, we have used $V_{\mathrm{sh}}, \boldsymbol{V}_{\mathrm{sw}}, \boldsymbol{B}$, and $\boldsymbol{n}$ provided by the CfA catalog instead of ipshocks.fi because of the generous error bars provided by the latter.

Figure 11 shows the phase-space distributions $f(v)$ as measured by $A C E /$ SWICS (red symbols) and $A C E / E P A M$ (blue symbols) conveniently transformed into the solar wind frame of reference at two different times before the arrival of the shocks. In such a transformation, we have assumed that particles propagate in the solar wind direction, and therefore we have overcorrected for the Compton-Getting effect. Instead of energy, in the two left columns of Figure 11, we express $f(v)$ as a function of $v / v_{\mathrm{esc}}$, where $v$ is the particle speed and $v_{\mathrm{esc}}=$ $U_{\text {up }} \sec \theta_{B n}$. The values of $v_{\text {esc }}$ are listed in each respective panel. In the first column of Figure 11, we have used the shock parameters from the $A C E$ Transient List, whereas in the second column, we have used the shock parameters from the ipshocks. fi catalog (with the exception of Figure 11(b2), where we have used the CfA parameters). Similarly, in the last two columns of Figure 11, we express $f(v)$ as a function of $v / W_{b}$, where $W_{b}$ has been computed using the shock parameters from the $A C E$ Transient List (third column) or the ipshocks.fi catalog (fourth column; with the exception of Figure 11(b4), where we have used the CfA shock parameters). The values of $W_{b}$ are listed in each respective panel. Whereas the first two columns of Figure 11 correspond to a 12 minute interval observed 
Table 2

Shock Speed, Shock Normal, and Upstream Solar Wind Velocities and Magnetic Field Vectors for the Selected Shocks Used to Compute $v_{\text {esc }}$ and $W_{b}$

\begin{tabular}{|c|c|c|c|c|c|c|c|c|c|c|}
\hline \multirow{2}{*}{$\begin{array}{l}\text { Shock Time } \\
\text { SC Year/Day/UT [doy] } \\
\text { (1) }\end{array}$} & \multicolumn{5}{|c|}{$A C E$ Transient List ${ }^{\mathrm{a}}$} & \multicolumn{5}{|c|}{ ipshocks.fi $^{\mathrm{b}}$} \\
\hline & $\begin{array}{l}V_{\text {sh }} \\
(2)\end{array}$ & $\begin{array}{c}V_{\mathrm{sw}}[\mathrm{RTN}] \\
\text { (3) }\end{array}$ & $\begin{array}{c}\hat{n}[\mathrm{RTN}] \\
(4)\end{array}$ & $\begin{array}{c}\boldsymbol{B}[\mathrm{RTN}] \\
(5)\end{array}$ & $\begin{array}{l}\theta_{U n} \\
(6)\end{array}$ & $\begin{array}{l}V_{\text {sh }} \\
(7)\end{array}$ & $\begin{array}{c}\boldsymbol{V}_{\mathrm{sw}}[\mathrm{GSE}] \\
(8)\end{array}$ & $\begin{array}{c}\hat{n}[\mathrm{GSE}] \\
(9)\end{array}$ & $\begin{array}{c}\boldsymbol{B}[\mathrm{GSE}] \\
\quad(10)\end{array}$ & $\begin{array}{l}\theta_{U n} \\
(11)\end{array}$ \\
\hline A $2001 / 023 / 10: 06: 36$ [023.4212] & 532 & $(411,-18,5)$ & $(0.90,0.22,-0.36)$ & $(-3.63,-0.26,1.22)$ & 27 & $582^{\mathrm{c}}$ & $(-417,18,7)^{\mathrm{c}}$ & $(-0.99,0.01,-0.09)^{\mathrm{c}}$ & $(3.50,0.60,1.30)^{\mathrm{c}}$ & 06 \\
\hline A $2002 / 198 / 15: 26: 14$ [198.6432] & 493 & $(410,-18,0)$ & $(0.90,0.38,-0.23)$ & $(4.85,-1.26,0.88)$ & 28 & 524 & $(-408,29,-3)$ & $(-0.93,-0.24,-0.29)$ & $(-6.34,-0.40,-0.59)$ & 24 \\
\hline A $2001 / 304 / 12: 53: 12[304.5369]$ & 458 & $(327,7,-9)$ & $(0.97,0.22,0.08)$ & $(3.49,-1.04,-0.59)$ & 13 & 455 & $(-330,-3,-10)$ & $(-0.98,0.04,-0.20)$ & $(-3.63,1.42,-0.43)$ & 10 \\
\hline A $2003 / 308 / 05: 59: 32$ [308.2497] & 779 & $(485,9,0)$ & $(0.98,-0.05,-0.17)$ & $(5.21,4.54,-0.36)$ & 11 & 491 & $(-490,11,2)$ & $(-0.47,0.82,-0.33)$ & $(5.39,-4.84,0.87)$ & 61 \\
\hline A $2001 / 118 / 04: 31: 59$ [118.1889] & 905 & $(460,38,5)$ & $(0.97,0.25,0.07)$ & $(0.45,-6.20,-2.52)$ & 10 & 992 & $(-468,-38,4)$ & $(-0.98,-0.20,0.01)$ & $(-1.07,8.10,-3.48)$ & 07 \\
\hline
\end{tabular}

Notes.

${ }^{\text {a }}$ Normal vectors provided by www.ssg.sr.unh.edu/mag/ace/ACElists/obs_list.html, and $V_{\text {sw }}$ and $B$ averaged upstream of the shock.

b Normal directions, $V_{\mathrm{sw}}$, and $B$ provided by ipshocks.fi/ (Kilpua et al. 2015).

${ }^{\mathrm{c}}$ Normal directions, $V_{\mathrm{sw}}$, and $B$ obtained from the CfA shock catalog at www.cfa.harvard.edu/shocks/. 

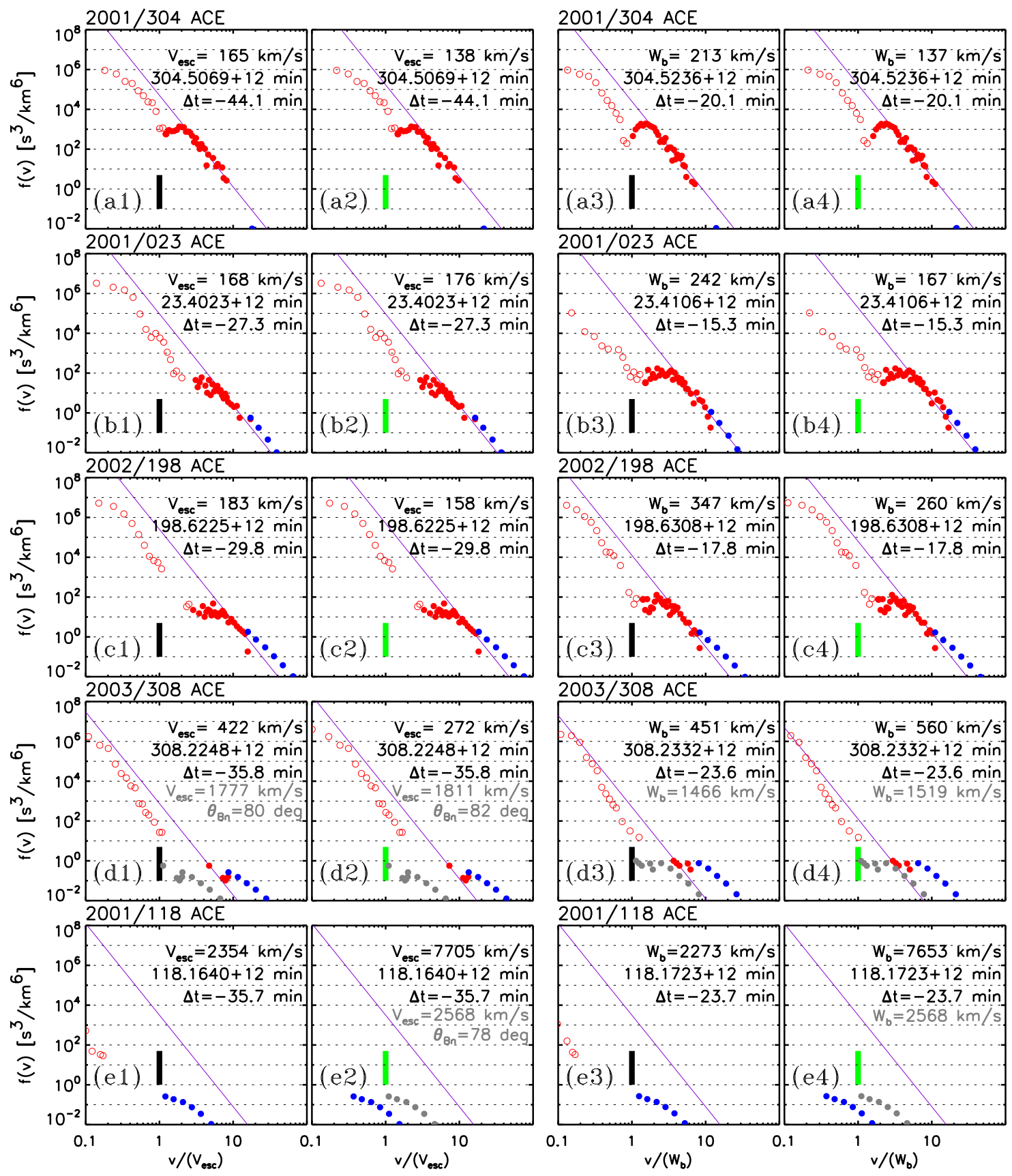

Figure 11. Two left columns: phase-space distribution functions in the solar wind frame as measured by ACE/SWICS (red symbols) and ACE/EPAM/LEMS120 (blue symbols) as a function of $v / v_{\text {esc }}$ measured in a 12 minute interval $\sim 30$ minutes before the shock arrival and using shock parameters from the $A C E$ Transient List (first column) and the ipshocks.fi catalog (second column) to compute $v_{\text {esc }}$ (with the exception of the shock on 2001/023, where the CfA parameters have been used). Two right columns: phase-space distribution functions in the solar wind frame as measured by ACE/SWICS (red symbols) and ACE/EPAM/LEMS120 (blue symbols) as a function of $v / W_{b}$ measured in a 12 minute interval just upstream of the shock and using shock parameters from the $A C E$ Transient List (third column) and the ipshocks.fi catalog (fourth column) to compute $W_{b}$ (with the exception of the shock on 2001/023, where the CfA parameters have been used).

$\sim 30$ minutes before the shock arrival, the last two columns correspond to a 12 minute interval observed before the arrival of the shock (the next 12 minute interval already encompasses some time downstream of the shock). Figure 11 shows that the use of the different shock parameters to compute $v_{\mathrm{esc}}$ or $W_{b}$ does not entail significant differences in the final shape of the $f(v)$ spectra. Similarly, the differences when scaling the particle speeds $v$ using either $v_{\text {esc }}$ or $W_{b}$ are minimal because of the small values of $\theta_{U n}$ (Table 2). The largest difference between $v_{\text {esc }}$ and $W_{b}$ is a factor of 2 observed for the shock on 2003/308 when using ipshocks.fi parameters because $\boldsymbol{U}$ departs the most from the normal direction (with an exceptionally large value of $\theta_{U n}=61^{\circ}$ ).

Figures 11(a1)-(a4) correspond to the energy spectra observed prior to the arrival of the shock on day 2001/304 (Section 2.2). A significant peak in the ST regime develops around $v / v_{\text {esc }} \sim 2$. The speed at which the ST portion of the spectrum (filled red symbols) increases is approximately $v=U_{\text {up }} \sec \theta_{B n}$, or $v=W_{b}$ (indicated by the vertical bars in Figure 11). The shock on 2001/304 corresponds, on average, to a quasi-parallel weak shock propagating into a region where amplification of waves is inhibited (such as in an ICME) and 
the intensity of shock-accelerated high-energy particles is not abundant enough to efficiently amplify enough waves. Therefore, the conditions were appropriate for the development of an extended ESP upstream region (observed for $\sim 12 \mathrm{hr}$ before the shock arrival; Figure 5(a)), the presence of anisotropic fieldaligned beams upstream of the shock (Figure 5(q)), and the development of an energy spectrum that is consistent with predicted upstream energy spectra, where peak energy gain occurs for upstream particle speeds $v / v_{\text {esc }} \sim 2$ when an ambient particle distribution is adiabatically accelerated at a structureless oblique shock under weak scattering conditions (e.g., Decker 1983).

Under more general conditions (i.e., oblique shocks propagating in a nominal solar wind where amplification of waves is not inhibited), upstream ST particle increases are not so extended in distance, and anisotropies are usually weaker. For the event on 2001/023, the ST enhancement was observed first at high energies starting $\sim 4 \mathrm{hr}$ before the shock arrival (Section 2.1.1). Figure 2 shows that the energy spectra populated first at high energies until reaching a bump with peak intensities around $\sim 6-10 \mathrm{keV}$. The energy spectra shown in Figures 11(b1) and (b2) correspond to the energy spectrum in Figure 2(d) but transformed into the solar wind frame and scaled by $v_{\text {esc }}$, where we can infer the development of a peak in the ST energy regime. It is not until a few minutes before the shock arrival when the bump in the energy spectra is clearly noticeable (Figures 11(b3), 11(b4), and 2(2)), but with an extended peak over the range $2 \lesssim v / W_{b} \lesssim 4$ broader than the peak in the case of the shock on 2001/304. In the 2001/023 event, the streaming of particles with $v / v_{\text {esc }} \lesssim 2$ may have been hindered by scattering processes occurring close to the shock, impeding both the formation of a clear field-aligned beam (Figure 1(q)) and the abundant arrival of low-energy particles at the spacecraft. In order to reach the spacecraft before the arrival of the shock, particles have to overcome the scattering effects, hence the more extended and energetic peak in the spectra in Figures 11(b3) and (b4) with respect to that in Figures 11(a3) and (a4). Scattering processes also favor the interaction of the particles with the shock and hence the more energetic ESP event.

The ST enhancement for the shock on 2002/198 also started at high energies but just $\sim 1 \mathrm{hr}$ before the shock passage (Section 2.1.2). Figures 11(c1) and (c2) correspond to the spectrum in Figure 4(c) where the ST population first started to increase at high energies. Figures $11(\mathrm{c} 3)$ and (c4) correspond to Figure 4(d) and show that the ST spectrum had an extended maximum at $2 \lesssim v / W_{b} \lesssim 4$. The ST energy spectra started to increase at speeds larger than $v_{\text {esc }}$. The compact and brief intensity increase in front of the shock on 198/2002 suggests that the particle intensities in this ESP event were shaped by the upstream depressed magnetic field and density region (indicated by arrows in Figures 3(1)-(n)), which was responsible for the confinement of low-energy particles near the shock front, whereas only high-energy $(\gtrsim 50 \mathrm{keV})$ particles were able to flow away from the shock. This depressed region may also result from the effect produced by the ESP particles themselves (Lario \& Decker 2002; Lario et al. 2015).

Intensity peaks upstream of the shocks in panels (a), (b), and (c) in Figure 11 at proton speeds $\sim 2-4 v_{\text {esc }}$ could originate from, among other processes, specular reflection or adiabatic mirror reflection of particles incident from upstream, or by transmission upstream of downstream particles (e.g., Balogh \&
Treuman 2014, and references therein). Specular reflection, which is the reversal of the particle velocity component normal to the shock surface at a single interaction with the cross-shock potential within the ramp of a structured shock, enables the particle to escape back upstream or transmit through the shock, depending on the incidence angle of the particle, that for particles tied to the magnetic field depends on $\theta_{B n}$. Adiabatic mirroring, which refers to acceleration and reflection of particles at oblique shocks, involves the reversal of an incident particle velocity parallel to the upstream magnetic field and an increase in its magnitude so that $v_{\|}>v_{\text {esc }}$. The parallel speed gained by particles adiabatically reflected at the shock front is approximately $\sim 2 v_{\text {esc }}$ (e.g., Terasawa 1979). Orbits of particles reflected adiabatically typically cross the shock (which can be treated as structureless for ions with gyroradii much larger than the shock thickness) many times during the reflection processes, drifting along the motional electric field as they do so, thereby gaining the parallel energy needed to escape the shock. Finally, heated and accelerated ions from the downstream region of the shock can escape back upstream if their parallel velocity exceeds that of the field along the shock surface, e.g., due to further acceleration in the downstream plasma or rearrangement of their perpendicular and parallel velocity components due to pitch-angle scattering. Due to the relatively low temporal, and therefore spatial, resolution of the available particle data in our study, we are unable to resolve the effects of the shock structure in producing the observed upstream peaks of the energy spectra at specific values of a few times $v_{\text {esc }}$. The three processes described above may contribute to the different extents of the observed upstream populations in the first three shocks discussed here, and we have just described the upstream particle spectra mainly according to the kinematic constraint implied by the average shock geometry and the range of $v_{\text {esc }}$ or $W_{b}$ values implied from this geometry.

Finally, the lack of observed ST particles upstream of the shocks on 2003/308 and 2001/118 (Section 2.3) suggests elevated values of $v_{\text {esc }}$ or $W_{b}$ or, equivalently, a more perpendicular configuration for these two shocks. For the shock on $2003 / 308$, the values of $\theta_{B n}$ at $A C E$ listed in Table 1 range from $22^{\circ}$ (although with generous error bars) to $43^{\circ}$. Note that the uncertainties of $V_{\text {sh }}$ provided by ipshocks.fi would translate into a broad range of values for $v_{\text {esc }}$, whereas in Figure 11(d2), we have just considered the central value of $V_{\text {sh }}$ provided by the catalogs without error bars. Figures 11(d1)(d4) show that the particle increase was observed just at the high-energy end of $A C E / \mathrm{SWICS}$ and at $A C E / \mathrm{EPAM} /$ LEMS120, and that corresponds to speeds well above the computed values of $v_{\text {esc }}$ or $W_{b}$. Therefore, we suspect that this shock was more perpendicular than the results of the shock parameters listed in Table 1 . In fact, if we increase $W_{b}$ or $v_{\mathrm{esc}}$ (or, equivalently, increase $\theta_{B n}$ when keeping $U_{\text {up }}$ constant), the energies from which we observe the intensity enhancement scale down to the value $v / v_{\text {esc }}=1$ or $v / W_{b}=1$ (gray symbols in Figures 11(d1)-(d4)), implying values of $\theta_{B n}$ close to $\sim 80^{\circ}$. The gap between the thermal population (open red symbols) and the high-energy enhanced populations (filled symbols) observed upstream of the shock on 2003/308 suggests that low-energy $(\lesssim 10 \mathrm{keV})$ particles were not able to escape from the shock vicinity. Therefore, in our distinction between thermal and ST regimes, the gap indicates that low-energy ST particles were not able to run away from the shock. One might argue that the true ST upstream population starts at the 
energies at which upstream particle enhancements are observed. However, the flattening or even rollover of the energy spectra in the low-energy end of $A C E /$ EPAM observed prior to the shock arrival (Figures 8(a)-(e)) casts some doubts on the existence of upstream ST particles (at least above the sensitivity of the instruments and as per our definition of the ST regime, which is independent of either the shock parameters or the facility of the particles to escape from the shock vicinity).

Similarly, the shock parameter catalogs (Table 1) indicate that the shock on 2001/118 was perpendicular at $A C E$ (with $\theta_{B n}>86^{\circ}$ ). This translates into large values of $W_{b}$ or $v_{\text {esc }}$. In fact, for the parameters provided by ipshocks.fi, it is necessary to reduce $W_{b}$ or $v_{\text {esc }}$ (or, equivalently, $\theta_{B n}$ to $\sim 78^{\circ}$ ) to agree with the energy at which we start observing upstream particles (gray symbols in Figures 11(e2) and (e4)), whereas for the $A C E$ Transient List parameters, the values of $\boldsymbol{B}$ and $\hat{n}$ in Table 2 already provide $\theta_{B n}=79^{\circ}$. The perpendicularity of the shock on 2001/118 implies that ST particles were not observed upstream of the shock. The shock catalogs also indicate that the shock on $2001 / 118$ was more oblique at Wind $\left(\theta_{B n} \sim 48^{\circ}\right)$ than at $A C E$, suggesting that particles could escape from the shock vicinity easier at Wind than at $A C E$. The easier escape of particles at Wind would have translated into the observation of a small bump in the spectra just prior to the shock passage (Figure 10(1)).

In Figure 11, we also analyze whether the common spectrum $v^{-5}$ suggested by Fisk \& Gloeckler $(2012,2014)$ was observed in our events. The solid purple lines in Figure 11 indicate the slope $v^{-5}$. Within the variety of spectra profiles observed upstream of the shocks, the slope $v^{-5}$ is only partially found over very limited energy ranges within the ST regime. By contrast, Figure 12 shows the energy spectra of $f(v)$ a few minutes after the shock passage where, according to Fisk \& Gloeckler (2014), the $v^{-5}$ spectra are more easily developed. For the purpose of comparing our observations with the results of Fisk \& Gloeckler $(2012,2014)$, we have scaled the speeds with the value of $V_{\mathrm{sw}}$ averaged over the time interval when the energy spectra in Figure 12 were measured (listed in the different panels). The solid purple lines in Figure 12 indicate an $\sim v^{-5}$ spectra. For most of the events, the downstream spectra right after the shock passage show a smooth transition from the heated thermal core population to the ST particles to high energies, regardless of the properties of the ST upstream population and shock parameters. A least-squares fit of an $f \propto v^{-\gamma}$ function to the ST ACE/SWICS data (filled red symbols in Figure 11) over the velocity interval $1<v / V_{\text {sw }}<$ 9 has been performed (note that for the event on 2001/118, the ST population detected by $A C E / S W I C S$ is restricted to large speeds because of the elevated temperature and plasma speed registered downstream of this shock (Figures 9(c)-(e)), and we have limited the power-law fit to the interval $1.6<v / V_{\text {sw }}<$ 4). The values of the spectral index $\gamma$ are listed in the respective panels. They are consistently below 5 , although within the error bars, they agree with $\gamma \sim 5$. The overcorrection applied to the transformation from spacecraft to solar wind frames may translate to lower values of $f(v)$ at lower speeds, and hence the consistent values below 5 . We also note that over certain speed intervals, especially at the higher-energy end, the agreement between the slope $v^{-5}$ and measurements is excellent, but not over the whole interval of measured speeds. Therefore, the
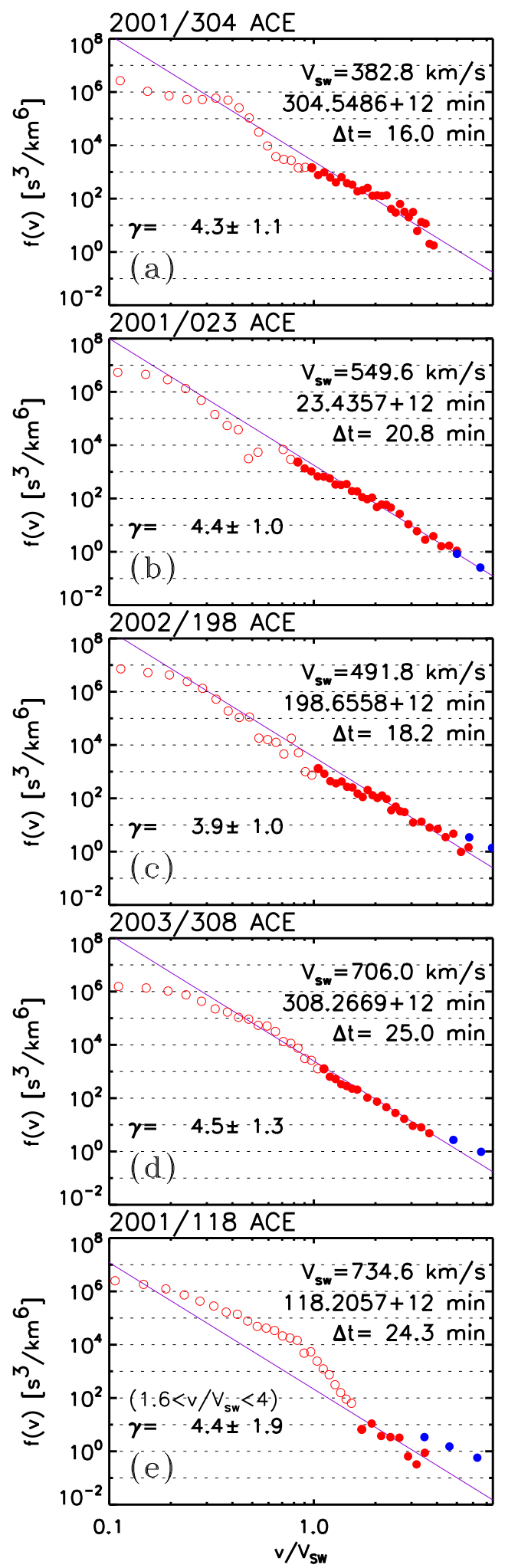

Figure 12. Downstream phase-space distribution functions in the solar wind frame as measured by $A C E /$ SWICS (red symbols) and $A C E / E P A M /$ LEMS120 (blue symbols) as a function of $v / V_{\text {sw }}$. The open red symbols indicate data points in the thermal energy regime, whereas the filled red symbols are in the ST regime. The purple lines indicate a dependence $f(v) \propto v^{-5}$. The index $\gamma$ in each panel has been obtained from a least-squares fit $f(v) \propto v^{-\gamma}$ to the ST ACE/SWICS data points over the interval $1<$ $v / V_{\mathrm{sw}}<9$. 
pump mechanism proposed by Fisk \& Gloeckler (2012), if present, must be efficient at lower energies and verifiable only downstream of the shocks. We should indicate that for the range of values for the density compression ratios $r_{n}$ of the shocks listed in Table 1, quasi-linear diffusive shock acceleration theory (Lee 1983) predicts power laws $v^{-\sigma}$ with slopes $\sigma=3 r_{n} /\left(r_{n}-1\right)$ that are in the range between -4 and -6 (including $v^{-5}$ ) observed downstream of the shock.

\section{Conclusions}

From the study of the five ESP events presented here, we have tried to synthesize the different types of evolution that the ST population exhibits around the passage of shocks near 1 au. At one extreme, this evolution ranges from velocity-dispersed onsets with energy spectra that fill gradually from high to low energies, developing into a spectrum with a peak around a few $\mathrm{keV}$ observed just before the arrival of the shock (e.g., 2001/304, 2001/023). At the other extreme, there is a complete absence of ST particles upstream of the shock (e.g., $2001 / 118$ ) or even sudden particle increases associated with plasma structures formed upstream of the shock (e.g., 2002/ 198). We wish to stress that the ESP events studied here are by no means representative of the whole variety of events that can be observed. The relationship between the shock parameters and the ST proton evolution is not unambiguous, since the shock parameters may differ using different computational methods or local single-point information from nearby spacecraft (Table 1). Moreover, particles in an ESP event might not only consist of those particles accelerated locally at the portion of the shock intercepted by the spacecraft, but most likely they might also include particles accelerated remotely at other portions of the shock front or at distant downstream locations. Irregular magnetic field structures upstream of the shock, as well as ripples on the shock front, may produce a magnetic connection between the spacecraft and distant regions of the shock front where particle acceleration may be more efficient than at the portion of the shock front intercepted by the spacecraft (Decker 1990; Giacalone 2005; Johlander et al. 2016). Therefore, the local observation of a shock (with a given set of shock parameters) is not necessarily correlated with the particle signatures, which may originate from other regions of the shock front (e.g., Giacalone \& Neugebauer 2008).

It has been suggested that ST particles may serve as "seed" particles to the mechanisms of particle acceleration to higher energies (e.g., Thomsen 1985; Mason 2000). However, in our events, the ST enhancements seen upstream of the shocks are very limited in time prior to the shock arrival and completely absent in the case of perpendicular shocks. The lack of ST intensities far upstream of the shock challenges the existence of STs as a seed population for particle acceleration, at least during the passage of the shocks at $1 \mathrm{au}$, and it indicates that solar wind thermal particles might be the predominant source of accelerated particles. It is possible that ST intensities remain below the sensitivity of instruments such as $A C E /$ SWICS or the background intensity level of Wind/3DP prior to the passage of the shocks. Instruments with better sensitivity are necessary to determine whether ST intensities upstream of the shock can explain the particle enhancements seen at shocks. Instrumental limitations have restricted most of the ST studies to long-term averages, where the precise contribution of ST populations to the particular processes of particle acceleration at shocks and downstream regions cannot be detailed on an event-by-event basis. However, these undetectable low intensities would require a very efficient acceleration mechanism. We do not exclude the possibility that traces of preexistent ST material upstream of the shock may participate in the process of particle acceleration, but the detailed evolution of the ST population around the passage of shocks at 1 au does not allow us to precisely assess the contribution of this material.

It is possible that the currently unknown dependence of the ST population on heliocentric distance allows a more abundant ST population close to the Sun. The IP shocks that start propagating close to the Sun might find an environment rich in ST particles. Accumulation of particles in the downstream region of the shocks throughout their transit from the Sun to 1 au shapes the final properties of the ESP events at $1 \mathrm{au}$. On the other hand, continuous acceleration of thermal solar wind particles may also provide the observed energy spectra downstream of the shocks, whereas traces of ST heavy ions may explain the ion composition abundances measured in ESP events (Giacalone 2017). Future inner heliospheric missions such as the Parker Solar Probe and Solar Orbiter, as well as the Interstellar Mapping and Acceleration Probe at L1, may provide answers regarding the contribution of ST populations to the processes of particle acceleration during the transit of IP shocks from the Sun to $1 \mathrm{au}$.

Data used in this paper can be downloaded from www.srl. caltech.edu/ACE/ASC/ and sprg.ssl.berkeley.edu/wind3dp/ data/wi/3dp/. We have used Wind/3DP data analysis software available at https://github.com/lynnbwilsoniii/wind_3dp_ pros. We acknowledge all the teams of the science instruments for making available their data used in this paper. We would like to thank the anonymous reviewer for the remarks. This paper uses data from the Heliospheric Shock Database, generated and maintained at the University of Helsinki (ipshocks.fi); the CfA Interplanetary Shock Database, generated and maintained at the Harvard-Smithsonian Center for Astrophysics (www.cfa.harvard.edu/shocks/); and the $A C E$ Lists of Disturbances and Transients (www.ssg.sr.unh.edu/ mag/ace/ACElists/obs_list.html). This work is supported by NASA-HGI grant NNX16AF73G and NASA/LWS grant NNX15AD03G. D.L. acknowledges the support from NASA Program NNH17ZDA001N-LWS. E.C.R. and D.L. acknowledge NASA support under $A C E$ grant NNX10AT75G.

\section{ORCID iDs}

D. Lario (1) https://orcid.org/0000-0002-3176-8704

R. F. Wimmer-Schweingruber (i) https://orcid.org/0000-00027388-173X

L. B. Wilson, III (iD https://orcid.org/0000-0002-4313-1970

\section{References}

Balogh, A., \& Treuman, R. A. 2014, Physics of Collisionless Shocks, Space Plasma Shock Waves (New York: Springer)

Berger, L. 2008, PhD thesis, Christian-Albrechts-Universität zu Kiel

Blanco-Cano, X., Kajdič, P., Aguilar-Rodríguez, E., et al. 2016, JGRA, 121, 992

Bryant, D. A., Cline, T. L., Desai, U. D., \& McDonald, F. B. 1962, JGR, 67,4983

Burgess, D. 1995, in Introduction to Space Physics, ed. M. G. Kivelson \& C. T. Russell (Cambridge: Cambridge Univ. Press), 129

Decker, R. B. 1983, JGR, 88, 9959

Decker, R. B. 1990, JGR, 95, 11993

Desai, M., \& Giacalone, J. 2016, LRSP, 13, 3 
Desai, M. I., Dayeh, M. A., \& Ebert, R. W. 2016, in AIP Conf. Ser. 1720, Solar Wind 14: Proceedings of the Fourteenth International Solar Wind Conference, ed. L. Wang et al. (Melville, NY: AIP), 060002

Fisk, L. A., \& Gloeckler, G. 2012, SSRv, 173, 433

Fisk, L. A., \& Gloeckler, G. 2014, JGRA, 119, 8733

Frank, L. A. 1970, JGR, 75, 707

Giacalone, J. 2005, ApJ, 624, 765

Giacalone, J. 2012, ApJ, 761, 28

Giacalone, J. 2017, JPhCS, 900, 012008

Giacalone, J., Burgess, D., Schwartz, S. J., \& Ellison, D. C. 1993, ApJ, 402,550

Giacalone, J., \& Neugebauer, M. 2008, ApJ, 673, 629

Gloeckler, G., Cain, J., Ipavich, F. M., et al. 1998, SSRv, 86, 497

Gloeckler, G., Geiss, J., Balsiger, H., et al. 1992, A\&AS, 92, 267

Gloeckler, G., Roelof, E. C., Ogilvie, K. W., \& Berdichevsky, D. B. 1995, SSRv, 72, 321

Gold, R. E., Krimigis, S. M., Hawkins, S. E., III, et al. 1998, SSRv, 86, 541

Gosling, J. T. 1983, SSRv, 34, 113

Gosling, J. T., Asbridge, J. R., Bame, S. J., et al. 1981, JGR, 86, 547

Gosling, J. T., Asbridge, J. R., Bame, S. J., Paschmann, G., \& Sckopke, N. 1978, GeoRL, 5, 957

Gosling, J. T., Bame, S. J., Feldman, W. C., et al. 1984, JGR, 89, 5409

Gurnett, D. A., \& Bhattacharjee, A. 2005, in Introduction to Plasma Physics, ed. D. A. Gurnett \& A. Bhattacharjee (Cambridge: Cambridge Univ. Press), 462

Haggerty, D. K., Roelof, E. C., Ho, G. C., \& Gold, R. E. 2006, AdSpR, 38, 995 Johlander, A., Schwartz, S. J., Vaivads, A., et al. 2016, PhRvL, 117, 165101

Kajdič, P., Hietala, H., \& Blanco-Cano, X. 2017, ApJL, 849, L27

Kilpua, E. K. J., Lumme, E., Andreeova, K., Isavnin, A., \& Koskinen, H. E. J. 2015, JGRA, 120, 4112

Koval, A., \& Szabo, A. 2008, JGRA, 113, A10110

Lanzerotti, L. J., Gold, R. E., Anderson, K. A., et al. 1992, A\&AS, 92, 349

Lario, D., Berger, L., Wilson, L. B., III, et al. 2018, JPhCS, 1100, 012014

Lario, D., \& Decker, R. B. 2002, GeoRL, 29, 1393

Lario, D., Decker, R. B., Livi, S., et al. 2005a, JGRA, 110, A09S11

Lario, D., Decker, R. B., Roelof, E. C., Reisenfeld, D. B., \& Sanderson, T. R. 2004, JGRA, 109, A01107
Lario, D., Decker, R. B., Roelof, E. C., \& Viñas, A.-F. 2015, ApJ, 813, 85

Lario, D., Ho, G. C., Decker, R. B., et al. 2003, in AIP Conf. Proc. 679, Solar Wind Ten 679, ed. M. Velli, R. Bruno, \& F. Malara (Melville, NY: AIP), 640

Lario, D., Hu, Q., Ho, G. C., et al. 2005b, in Solar Wind 11/SOHO 16 , Connecting Sun and Heliosphere 592, ed. B. Fleck, T. H. Zurbuchen, \& H. Lacoste (Noordwijk: ESA), 81

Lario, D., Kwon, R.-Y., Riley, P., \& Raouafi, N. E. 2017, ApJ, 847, 103

Lario, D., Sanahuja, B., \& Heras, A. M. 1998, ApJ, 509, 415

Lee, M. A. 1983, JGR, 88, 6109

Lee, M. A. 2005, ApJS, 158, 38

Lepping, R. P., Acũna, M. H., Burlaga, L. F., et al. 1995, SSRv, 71, 207

le Roux, J. A., \& Webb, G. M. 2012, ApJ, 746, 104

Lin, R. P., Anderson, K. A., Ashford, S., et al. 1995, SSRv, 71, 125

Marhavilas, P. K., Malandraki, O. E., \& Anagnostopoulos, G. C. 2015, P\&SS, 117, 192

Mason, G. M. 2000, in AIP Conf. Proc. 528, Acceleration and Transport of Energetic Particles Observed in the Heliosphere, ed. R. A. Mewaldt, M. Miller, J. R. Jokipii et al. (Melville, NY: AIP), 234

McComas, D. J., Bame, S. J., Barker, P., et al. 1998, SSRv, 86, 563

Ogilvie, K. W., Chornay, D. J., Fritzenreiter, R. J., et al. 1995, SSRv, 71, 55

Ogilvie, K. W., Geiss, J., Gloeckler, G., Berdichevsky, D., \& Wilken, B. 1993, JGR, 98, 3605

Smith, C. W., L'Heureux, J., Ness, N. F., et al. 1998, SSRv, 86, 613

Szabo, A. 1994, JGR, 99, 14

Szabo, A., Lepping, R. P., Merka, J., Smith, C. W., \& Skoug, R. M. 2001, in Proc. First Solar Orbiter Workshop, Solar Encounter, ed. B. Battrick \& H. Sawaya-Lacoste (Noordwijk: ESA), 383

Terasawa, T. 1979, P\&SS, 27, 193

Thomsen, M. F. 1985, GMS, 35, 253

Tokar, R. L., Gary, S. P., Gosling, J. T., et al. 2000, JGR, 105, 7521

Torsti, J., Riihonen, E., \& Kocharov, L. 2004, ApJL, 600, L83

van Nes, P., Roelof, E. C., Reinhard, R., Sanderson, T. R., \& Wenzel, K.-P. 1985, JGR, 90, 3981

Wilson, L. B., III, Cattell, C. A., Kellogg, P. J., et al. 2009, JGRA, 114, A10106

Wilson, L. B., III, Cattell, C. A., Kellogg, P. J., et al. 2010, JGRA, 115, A12104

Zank, G. P., Hunana, P., Mostafavi, P., et al. 2015, ApJ, 814, 137

Zank, G. P., Li, G., Florinski, V., et al. 2006, JGRA, 111, A06108 